\title{
Transport coefficients of a relativistic plasma
}

\author{
O. J. Pike ${ }^{1, *}$ and S. J. Rose $\mathrm{R}^{1,2}$ \\ ${ }^{1}$ Blackett Laboratory, Imperial College, London SW7 2AZ, United Kingdom \\ ${ }^{2}$ Clarendon Laboratory, University of Oxford, Oxford OX1 3PU, United Kingdom
}

(Received 2 November 2015; revised manuscript received 22 April 2016; published 24 May 2016)

\begin{abstract}
In this work, a self-consistent transport theory for a relativistic plasma is developed. Using the notation of Braginskii [S. I. Braginskii, in Reviews of Plasma Physics, edited by M. A. Leontovich (Consultants Bureau, New York, 1965), Vol. 1, p. 174], we provide semianalytical forms of the electrical resistivity, thermoelectric, and thermal conductivity tensors for a Lorentzian plasma in a magnetic field. This treatment is then generalized to plasmas with arbitrary atomic number by numerically solving the linearized Boltzmann equation. The corresponding transport coefficients are fitted by rational functions in order to make them suitable for use in radiation-hydrodynamic simulations and transport calculations. Within the confines of linear transport theory and on the assumption that the plasma is optically thin, our results are valid for temperatures up to a few MeV. By contrast, classical transport theory begins to incur significant errors above $k_{B} T \sim 10 \mathrm{keV}$, e.g., the parallel thermal conductivity is suppressed by $15 \%$ at $k_{B} T=20 \mathrm{keV}$ due to relativistic effects.
\end{abstract}

DOI: 10.1103/PhysRevE.93.053208

\section{INTRODUCTION}

Thermonuclear burn in inertial confinement fusion (ICF) is predicted to involve the most extreme temperatures, densities, and pressures ever produced in the laboratory [1]. It is hoped that temperatures indicative of a relativistic electron distribution $\left(k_{B} T \sim 10 \mathrm{keV}\right.$ and above) are reached in deuteriumtritium targets on the National Ignition Facility [2,3]. The development of other ICF schemes, such as fast ignition [4] and shock ignition [5], is also predicated on achieving such temperatures. Prospective tritium-poor or pure deuterium inertial fusion schemes are expected to involve even higher temperatures $\left(k_{B} T \sim 100 \mathrm{keV}\right)$ [6].

Transport processes, particularly electron thermal conduction, are important for the formation of the hot spot in ICF and the dynamics of the subsequent propagating burn wave [7]. Although these systems are conventionally unmagnetized, recently both imposed [8] and self-generated [9] electromagnetic fields have been studied in ICF-like plasmas, and these are likely to have profound effects on electron transport.

There is therefore a growing need for a complete transport theory for plasmas, which fully accounts for the effects of special relativity. In a previous paper [10], we derived the dynamical friction and diffusion coefficients for a relativistic plasma in the same form as those of Trubnikov [11]. In the present work, we use these results to calculate the transport coefficients of a plasma in which the electron distribution is relativistic.

The subject of relativistic transport has been studied in the past by various authors. McBride and Pytte [12] first determined the electron conductivity of a magnetized plasma using the collision operator of Beliaev and Budker [13] to lowest order in the relativistic correction $\left(v^{2} / c^{2}\right)$. Dzhavakhishvili and Tsintsadze [14] soon after calculated the transport coefficients of an ultrarelativistic plasma $\left(k_{B} T_{e} \gg\right.$ $m_{e} c^{2}$ ) using a similar approach to that of Braginskii [15].

\footnotetext{
*o.pike11@imperial.ac.uk
}

Balescu et al. [16] determined the form of the thermal conductivity, along with the shear and bulk viscosities, for a relativistic plasma but did not provide numerical results. With reference to the early universe, van Erkelens and van Leeuwen [17] calculated the electrical conductivity of a pair plasma, including the effects of the interaction with a radiation field. (A similar analysis was performed later by Kremer and Patsko [18].) Braams and Karney [19,20], using an expansion of the relativistic Fokker-Planck collision operator in spherical harmonics, derived the electrical conductivity of a nonmagnetized plasma with arbitrary ionic charge. Mohanty and Baral $[21,22]$ used a modified Chapman-Enskog analysis to calculate the cross-field transport coefficients, although their expression for the thermal conductivity diverges in the weak-field limit. Honda and Mima [23] derived the transport coefficients via an expansion in spherical harmonics, though they do not evaluate the cross-field terms explicitly and neglect electron-electron collisions.

To our knowledge, the only previous work which attempts to describe magnetized transport in a relativistic plasma, while including the effects of both electron-ion and electronelectron scattering, is that of Metens and Balescu [24]. Using an expansion in Hermite polynomials, they provide expressions for all components of the thermal and electrical conductivities. However, their results are inconsistent with those derived here, as well as those of other authors (e.g., electrical conductivity with Braams and Karney [20] and thermal conductivity with Honda and Mima [23]) and the magnitude of their relativistic corrections cannot be reconciled with the changes in the distribution function; significant corrections are given at mild temperatures, for which the classical and relativistic Maxwellian distributions are closely aligned.

For the transport theory developed in this work, we adopt the notation of Braginskii [15], whose relations are

$$
\begin{gathered}
e n \mathbf{E}=-\nabla p+\mathbf{j} \times \mathbf{B}+\boldsymbol{\alpha} \cdot \mathbf{j} / n e-n k_{B} \boldsymbol{\beta} \cdot \nabla T, \\
\mathbf{q}=-\boldsymbol{\kappa} \cdot \nabla T-\boldsymbol{\beta}^{\prime} \cdot \mathbf{j} k_{B} T / e,
\end{gathered}
$$


where $e$ is the elementary charge, $n$ is the electron density, $p$ is the scalar pressure, $k_{B}$ is the Boltzmann constant, $T$ is the electron temperature, $\mathbf{j}$ is the electric current, $\mathbf{q}$ is the total heat flow, $\mathbf{E}$ is the electric field strength, and $\mathbf{B}$ is the magnetic flux density. $\boldsymbol{\alpha}, \boldsymbol{\beta}$, and $\boldsymbol{\kappa}$ are the electrical resistivity, thermoelectric, and thermal conductivity tensors, respectively, whose relativistic forms this paper seeks to calculate. Finally, in the case of a nonrelativistic plasma,

$$
\boldsymbol{\beta}^{\prime}=\boldsymbol{\beta}+\frac{5}{2} \mathrm{l},
$$

where I is the unit diagonal second-order tensor.

This paper is structured as follows: Sec. II outlines the derivation of the relativistic Ohm's law and heat flow equation and presents the transport coefficients for a Lorentzian plasma in semianalytical form. This analysis is generalized to systems with arbitrary atomic number in Sec. III, for which the linearized Boltzmann equation is solved numerically. Such an approach is equivalent to an infinite expansion of the collision operator in Laguerre or Hermite polynomials, so long as the numerical grid is sufficiently fine [25]. In Sec. IV we provide rational function fits for the various transport coefficients, such that they may readily be used in transport calculations. Section $\mathrm{V}$ then follows with a discussion on these results, including the size of the relativistic corrections, and, finally in Sec. VI, we discuss the limits of applicability of this work.

\section{LORENTZIAN PLASMA: ANALYTICAL TREATMENT}

We begin our analysis with the Boltzmann equation for the electron population:

$$
\frac{\partial f_{e}}{\partial t}+\mathbf{v} \cdot \frac{\partial f_{e}}{\partial \mathbf{r}}-e(\mathbf{E}+\mathbf{v} \times \mathbf{B}) \cdot \frac{\partial f_{e}}{\partial \mathbf{u}}=\sum_{b} C^{e / b},
$$

where $\mathbf{u} \equiv \mathbf{p} / m_{e}$ is the momentum per species mass $m_{e}, \mathbf{v}=$ $\mathbf{u} / \gamma$ is the velocity (where $\gamma=\left(1+u^{2} / c^{2}\right)^{1 / 2}$ with $\left.u=|\mathbf{u}|\right)$, $f_{e}(\mathbf{r}, \mathbf{u}, t)$ is the electron distribution function as expressed in terms of position $\mathbf{r}$, momentum per unit mass $\mathbf{u}$ and time $t$, $C^{e / b}$ is the collision term, and $b$ represents all species present in the plasma. Here we are interested solely in electrons and ions; $b \in(e, i)$.

In order to solve the Boltzmann equation, an expansion of the distribution function and collision operator can be made in Cartesian tensors [26]. Under the assumption that the distribution is only weakly perturbed from equilibrium, the first two terms of this expansion are sufficient for an accurate description of transport:

$$
f(\mathbf{r}, \mathbf{u}, t)=f_{0}(\mathbf{r}, u, t)+\mathbf{f}_{1}(\mathbf{r}, u, t) \cdot \frac{\mathbf{u}}{u},
$$

as $\mathbf{f}_{1}$ describes the anisotropy in the system which leads to the current and heat flow. Substituting this truncated expansion into the Boltzmann equation [Eq. (4)] yields the $\mathbf{f}_{1}$ equation:

$$
\frac{\partial \mathbf{f}_{1}}{\partial t}+v \nabla f_{0}-\frac{e}{m_{e}} \mathbf{E} \frac{\partial f_{0}}{\partial u}-\frac{e}{\gamma m_{e}} \mathbf{B} \times \mathbf{f}_{1}=\mathbf{C}_{1},
$$

where $\mathbf{C}_{1}$ is the collision term and $v=|\mathbf{v}|$. (We have dropped the subscript $e$ from the distribution function $f$ for brevity.) It is also possible to derive in a similar manner an equation for the time evolution of $f_{0}$. However, in this work this is taken to be the relativistic Maxwellian, $f_{0}(\mathbf{r}, u, t)=f_{J}(u)$, with

$$
f_{J}(u)=\frac{n e^{-\gamma / \Theta}}{4 \pi c^{3} \Theta K_{2}(1 / \Theta)},
$$

and therefore invariant with respect to both $\mathbf{r}$ and $t$. Here $\Theta \equiv k_{B} T / m_{e} c^{2}$ is the reduced electron temperature, and $K_{v}$ is the $v$ th-order Bessel function of the second kind [27].

It is also possible to expand to higher orders and consider the time evolution of, e.g., $f_{2}$. However, in this analysis we set all higher order terms to zero: this corresponds to ignoring the effects of pressure anisotropy on the transport.

Our neglect of all higher order terms than $l=1$ in the expansion of the Boltzmann equation forms part of the diffusive approximation. The remaining step is to disregard the time derivative term, $\partial \mathbf{f}_{1} / \partial t$, in Eq. (6); we do so now. This allows us to find the steady-state solution of this equation.

In general, the collision term includes contributions from both electron-electron and electron-ion scattering, $\mathbf{C}_{1}=\mathbf{C}_{1}^{e / e}+\mathbf{C}_{1}^{e / i}$. However, in the Lorentz limit $(Z \rightarrow \infty)$, electron-electron collisions may be neglected; electron-ion collisions dominate for these systems as scattering scales strongly with charge $\left(\sim Z^{2}\right)$. Assuming the ions to be infinitely massive, $m_{i} \rightarrow \infty$, and at rest, $f_{i}(\mathbf{r}, \mathbf{u}, t)=\delta(\mathbf{u})$, the collision term then reduces to the simple form

$$
\mathbf{C}_{1}=-v_{e i} \mathbf{f}_{1},
$$

where

$$
v_{e i}=\frac{\Gamma^{e / i}}{u^{2} v}
$$

is the electron-ion collision frequency. The coefficient $\Gamma^{e / i}$ is given by

$$
\Gamma^{e / i}=\frac{Z n e^{4} \ln \Lambda^{e / i}}{4 \pi \epsilon_{0}^{2} m_{e}^{2}},
$$

where $Z$ is the atomic number of the ionic species, $\epsilon_{0}$ is the permittivity of free space, $\ln \Lambda^{e / i}$ is the electron-ion Coulomb logarithm, $n=n_{e}$, and we have assumed that the plasma is quasineutral: $Z n_{i}=n_{e}$. The $\mathbf{f}_{1}$ equation now reads

$$
v \nabla f_{0}-\frac{e}{\gamma m_{e}} \mathbf{B} \times \mathbf{f}_{1}-\frac{e}{m_{e}} \mathbf{E} \frac{\partial f_{0}}{\partial u}=-v_{e i} \mathbf{f}_{1} .
$$

Letting $\mathbf{B}=(0,0, B)$ and introducing the classical electron gyro-frequency $\omega=e B / m_{e}$, the components of $\mathbf{f}_{1}$ are given by

$$
\begin{aligned}
f_{1, x}= & \frac{u^{4} f_{J}}{u^{6} \omega^{2}+\left(\Gamma^{e / i} \gamma^{2}\right)^{2}}\left(-\Gamma^{e / i} \gamma^{2}\left\{\frac{\partial_{x} p}{p}+\left[\frac{\gamma}{\Theta}-\frac{K_{1}(1 / \Theta)}{\Theta K_{2}(1 / \Theta)}-4\right] \frac{\partial_{x} T}{T}+\left(\frac{e}{k_{B} T}\right) E_{x}\right\}\right. \\
& \left.+u^{3} \omega\left\{\frac{\partial_{y} p}{p}+\left[\frac{\gamma}{\Theta}-\frac{K_{1}(1 / \Theta)}{\Theta K_{2}(1 / \Theta)}-4\right] \frac{\partial_{y} T}{T}+\left(\frac{e}{k_{B} T}\right) E_{y}\right\}\right),
\end{aligned}
$$




$$
\begin{aligned}
f_{1, y}= & \frac{-u^{4} f_{J}}{u^{6} \omega^{2}+\left(\Gamma^{e / i} \gamma^{2}\right)^{2}}\left(u^{3} \omega\left\{\frac{\partial_{x} p}{p}+\left[\frac{\gamma}{\Theta}-\frac{K_{1}(1 / \Theta)}{\Theta K_{2}(1 / \Theta)}-4\right] \frac{\partial_{x} T}{T}+\left(\frac{e}{k_{B} T}\right) E_{x}\right\}\right. \\
& \left.+\Gamma^{e / i} \gamma^{2}\left\{\frac{\partial_{y} p}{p}+\left[\frac{\gamma}{\Theta}-\frac{K_{1}(1 / \Theta)}{\Theta K_{2}(1 / \Theta)}-4\right] \frac{\partial_{y} T}{T}+\left(\frac{e}{k_{B} T}\right) E_{y}\right\}\right),
\end{aligned}
$$

where $\partial_{x} \equiv \partial / \partial x, \partial_{y} \equiv \partial / \partial y$ and we have made use of the relations:

$$
\begin{gathered}
\partial_{x} f_{J}=\left\{\frac{\partial_{x} n}{n}+\left[\frac{\gamma}{\Theta}-\frac{K_{1}(1 / \Theta)}{\Theta K_{2}(1 / \Theta)}-3\right] \frac{\partial_{x} T}{T}\right\} f_{J}, \\
\frac{\partial f_{J}}{\partial u}=-\frac{u}{\gamma \Theta c^{2}} f_{J},
\end{gathered}
$$

as well as the equation of state for an ideal relativistic gas, $p=n k_{B} T$ [28]. Once the form of $\mathbf{f}_{1}$ is known, the electric current and total heat flow are calculated via integrations over momentum space [26]:

$$
\begin{gathered}
\mathbf{j}=-\frac{4 \pi e}{3} \int \mathbf{f}_{1} v u^{2} d u, \\
\mathbf{q}=\frac{4 \pi m_{e} c^{2}}{3} \int \mathbf{f}_{1}(\gamma-1) v u^{2} d u,
\end{gathered}
$$

which, in this case, yield

$$
\begin{gathered}
j_{x}=-\frac{4 \pi e}{3}\left(-\Gamma^{e / i}\left\langle u^{5,1}\right\rangle\left\{\frac{\partial_{x} p}{p}-\left[\frac{K_{1}(1 / \Theta)}{\Theta K_{2}(1 / \Theta)}+4\right] \frac{\partial_{x} T}{T}+\left(\frac{e}{k_{B} T}\right) E_{x}\right\}-\Gamma^{e / i}\left\langle u^{5,2}\right\rangle \frac{1}{\Theta} \frac{\partial_{x} T}{T}\right. \\
\left.+\omega\left\langle u^{8,-1}\right\rangle\left\{\frac{\partial_{y} p}{p}-\left[\frac{K_{1}(1 / \Theta)}{\Theta K_{2}(1 / \Theta)}+4\right] \frac{\partial_{y} T}{T}+\left(\frac{e}{k_{B} T}\right) E_{y}\right\}+\omega\left\langle u^{8,0}\right\rangle \frac{1}{\Theta} \frac{\partial_{y} T}{T}\right), \\
j_{y}=\frac{4 \pi e}{3}\left(\omega\left\langle u^{8,-1}\right\rangle\left\{\frac{\partial_{x} p}{p}-\left[\frac{K_{1}(1 / \Theta)}{\Theta K_{2}(1 / \Theta)}+4\right] \frac{\partial_{x} T}{T}+\left(\frac{e}{k_{B} T}\right) E_{x}\right\}+\omega\left\langle u^{8,0}\right\rangle \frac{1}{\Theta} \frac{\partial_{x} T}{T}\right. \\
\left.+\Gamma^{e / i}\left\langle u^{5,1}\right\rangle\left\{\frac{\partial_{y} p}{p}-\left[\frac{K_{1}(1 / \Theta)}{\Theta K_{2}(1 / \Theta)}+4\right] \frac{\partial_{y} T}{T}+\left(\frac{e}{k_{B} T}\right) E_{y}\right\}+\Gamma^{e / i}\left\langle u^{5,2}\right\rangle \frac{1}{\Theta} \frac{\partial_{y} T}{T}\right), \\
q_{x}=\frac{4 \pi m_{e} c^{2}}{3}\left(-\Gamma^{e / i}\left(\left\langle u^{5,2}\right\rangle-\left\langle u^{5,1}\right\rangle\right)\left\{\frac{\partial_{x} p}{p}-\left[\frac{K_{1}(1 / \Theta)}{\Theta K_{2}(1 / \Theta)}+4\right] \frac{\partial_{x} T}{T}+\left(\frac{e}{k_{B} T}\right) E_{x}\right\}-\Gamma^{e / i}\left(\left\langle u^{5,3}\right\rangle-\left\langle u^{5,2}\right\rangle\right) \frac{1}{\Theta} \frac{\partial_{x} T}{T}\right. \\
\left.+\omega\left(\left\langle u^{8,0}\right\rangle-\left\langle u^{8,-1}\right\rangle\right)\left\{\frac{\partial_{y} p}{p}-\left[\frac{K_{1}(1 / \Theta)}{\Theta K_{2}(1 / \Theta)}+4\right] \frac{\partial_{y} T}{T}+\left(\frac{e}{k_{B} T}\right) E_{y}\right\}+\omega\left(\left\langle u^{8,1}\right\rangle-\left\langle u^{8,0}\right\rangle\right) \frac{1}{\Theta} \frac{\partial_{y} T}{T}\right), \\
q_{y}=-\frac{4 \pi m_{e} c^{2}}{3}\left(\omega\left(\left\langle u^{8,0}\right\rangle-\left\langle u^{8,-1}\right\rangle\right)\left\{\frac{\partial_{x} p}{p}-\left[\frac{K_{1}(1 / \Theta)}{\Theta K_{2}(1 / \Theta)}+4\right] \frac{\partial_{x} T}{T}+\left(\frac{e}{k_{B} T}\right) E_{x}\right\}+\omega\left(\left\langle u^{8,1}\right\rangle-\left\langle u^{8,0}\right\rangle\right) \frac{1}{\Theta} \frac{\partial_{x} T}{T}\right. \\
\left.+\Gamma^{e / i}\left(\left\langle u^{5,2}\right\rangle-\left\langle u^{5,1}\right\rangle\right)\left\{\frac{\partial_{y} p}{p}-\left[\frac{K_{1}(1 / \Theta)}{\Theta K_{2}(1 / \Theta)}+4\right] \frac{\partial_{y} T}{T}+\left(\frac{e}{k_{B} T}\right) E_{y}\right\}+\Gamma^{e / i}\left(\left\langle u^{5,3}\right\rangle-\left\langle u^{5,2}\right\rangle\right) \frac{1}{\Theta} \frac{\partial_{y} T}{T}\right)
\end{gathered}
$$

where we have introduced the class of integral

$$
\left\langle u^{i, j}\right\rangle=\int_{0}^{\infty} \frac{f_{J}}{u^{6} \omega^{2}+\left(\Gamma^{e / i} \gamma^{2}\right)^{2}} u^{i+2} \gamma^{j} d u .
$$

Making $\mathbf{E}$ the subject of Eqs. (11), one may verify that the Braginskii formalism of Ohm's law [Eq. (1)] is satisfied in the relativistic case.

The corresponding transport coefficients $\varphi$ may be expressed in terms of components relative to the magnetic field vector $\mathbf{b}=\mathbf{B} /|\mathbf{B}|$ and driving force $\mathbf{s}$ by using

$$
\varphi \cdot \mathbf{s}=\varphi_{\|} \mathbf{b}(\mathbf{b} \cdot \mathbf{s})+\varphi_{\perp} \mathbf{b} \times(\mathbf{s} \times \mathbf{b}) \pm \varphi_{\lambda} \mathbf{b} \times \mathbf{s},
$$

where $\varphi \in\{\alpha, \beta, \kappa\}, \mathbf{s} \in\{\mathbf{E}, \nabla T\}$ and the negative sign applies only in the case $\varphi=\alpha$. This geometry is shown in Fig. 1 . In this notation, we find

$$
\alpha_{\perp}=\frac{3 n^{2} k_{B} T}{4 \pi \Gamma^{e / i}\left\langle u^{5,1}\right\rangle \Delta}
$$




$$
\begin{gathered}
\alpha_{\wedge}=\omega m_{e} n\left[\frac{3 n k_{B} T\left\langle\left\langle u_{5,1}^{8,-1}\right\rangle\right\rangle}{4 \pi\left(\Gamma^{e / i}\right)^{2} m_{e}\left\langle u^{5,1}\right\rangle \Delta}-1\right], \\
\beta_{\perp}=\frac{\left\langle\left\langle u_{5,1}^{5,2}\right\rangle\right\rangle+\left\langle\left\langle u_{8,-1}^{8,0}\right\rangle\right\rangle\left\langle\left\langle u_{5,1}^{8,-1}\right\rangle\right\rangle^{2}\left(\omega / \Gamma^{e / i}\right)^{2}}{\Theta \Delta}-\left[\frac{K_{1}(1 / \Theta)}{\Theta K_{2}(1 / \Theta)}+4\right], \\
\beta_{\wedge}=\frac{\omega\left\langle\left\langle u_{5,1}^{8,-1}\right\rangle\right\rangle\left[\left\langle\left\langle u_{8,-1}^{8,0}\right\rangle\right\rangle-\left\langle\left\langle u_{5,1}^{5,2}\right\rangle\right]\right]}{\Gamma^{e / i} \Theta \Delta},
\end{gathered}
$$

where $\left\langle\left\langle u_{i^{\prime}, j^{\prime}}^{i, j}\right\rangle\right\rangle \equiv\left\langle u^{i, j}\right\rangle /\left\langle u^{i^{\prime}, j^{\prime}}\right\rangle$ and $\Delta=1+\left(\omega\left\langle\left\langle u_{5,1}^{8,-1}\right\rangle\right\rangle / \Gamma^{e / i}\right)^{2}$. The parallel components of the transport coefficients may be found by taking the $\omega \rightarrow 0$ limit of the perpendicular components. [The component of the transport parallel to the magnetic field is independent of the magnitude of $\mathbf{B}$, as may be straightforwardly shown by taking the scalar product of the $\mathbf{f}_{1}$ equation [Eq. (10)] with B.]

The relativistic heat transfer equation can be deduced by substituting Eqs. (11) into (17). This also takes the same form as in classical theory [Eq. (2)], with the revised relation

$$
\boldsymbol{\beta}^{\prime}=\boldsymbol{\beta}+\left[\frac{K_{1}(1 / \Theta)}{\Theta K_{2}(1 / \Theta)}+4-\frac{1}{\Theta}\right] \mathrm{I},
$$

[cf. Eq. (3)] and the following thermal conductivity coefficients:

$$
\begin{aligned}
\kappa_{\perp}= & \frac{4 \pi \Gamma^{e / i}}{3 \Theta^{2}}\left\{\left(\left\langle u^{5,3}\right\rangle-\left\langle u^{5,2}\right\rangle\right)-\left(\left\langle u^{5,2}\right\rangle-\left\langle u^{5,1}\right\rangle\right) \frac{\left\langle\left\langle u_{5,1}^{5,2}\right\rangle\right\rangle+\left\langle\left\langle u_{8,-1}^{8,0}\right\rangle\right\rangle\left\langle\left\langle u_{5,1}^{8,-1}\right\rangle\right\rangle^{2}\left(\omega / \Gamma^{e / i}\right)^{2}}{\Delta}\right. \\
& \left.+\left(\left\langle u^{8,0}\right\rangle-\left\langle u^{8,-1}\right\rangle\right) \frac{\left\langle\left\langle u_{5,1}^{8,-1}\right\rangle\right\rangle\left(\omega / \Gamma^{e / i}\right)^{2}\left[\left\langle\left\langle u_{8,-1}^{8,0}\right\rangle\right\rangle-\left\langle\left\langle u_{5,1}^{5,2}\right\rangle\right]\right]}{\Delta}\right\}, \\
\kappa_{\wedge}= & \frac{4 \pi \omega}{3 \Theta^{2}}\left\{\left(\left\langle u^{8,1}\right\rangle-\left\langle u^{8,0}\right\rangle\right)-\left(\left\langle u^{8,0}\right\rangle-\left\langle u^{8,-1}\right\rangle\right) \frac{\left.\left\langle u_{5,1}^{5,2}\right\rangle\right\rangle+\left\langle\left\langle u_{8,-1}^{8,0}\right\rangle\right\rangle\left\langle\left\langle u_{5,1}^{8,-1}\right\rangle\right\rangle^{2}\left(\omega / \Gamma^{e / i}\right)^{2}}{\Delta}\right. \\
& \left.-\left(\left\langle u^{5,2}\right\rangle-\left\langle u^{5,1}\right\rangle\right) \frac{\left\langle\left\langle u_{5,1}^{8,-1}\right\rangle\right\rangle\left[\left\langle\left\langle u_{8,-1}^{8,0}\right\rangle-\left\langle\left\langle u_{5,1}^{5,2}\right\rangle\right\rangle\right]\right.}{\Delta}\right\} .
\end{aligned}
$$

\section{A. Limiting cases}

Equations (19)-(25) are the relativistic forms of Braginskii's transport coefficients for a Lorentzian plasma and can be shown to reduce to other known results in particular limits. We consider two such cases.

First, in the limit $\Theta \rightarrow 0$, these results reduce to those in the classical work of Epperlein [29]:

$$
\alpha_{\perp}=\frac{3 n^{2} k_{B} T}{4 \pi \Gamma^{e / i}\left\langle v^{5}\right\rangle \Delta},
$$

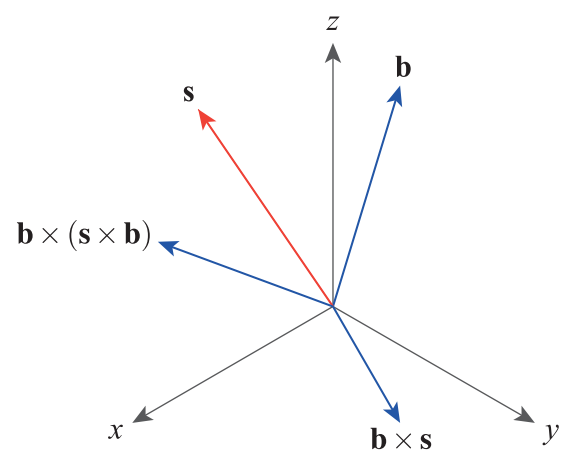

FIG. 1. The geometry used in our description of transport, as per Eq. (18). The tensorial transport coefficients are described by their components in the $\mathbf{b}\left({ }_{\|}\right), \mathbf{b} \times(\mathbf{s} \times \mathbf{b})\left({ }_{\perp}\right)$ and $\mathbf{b} \times \mathbf{s}\left({ }_{\wedge}\right)$ directions.

$$
\begin{gathered}
\alpha_{\wedge}=\omega m_{e} n\left[\frac{3 n k_{B} T\left\langle\left\langle v_{5}^{8}\right\rangle\right\rangle}{4 \pi\left(\Gamma^{e / i}\right)^{2} m_{e}\left\langle v^{5}\right\rangle \Delta}-1\right] \\
\beta_{\perp}=\frac{\left\langle\left\langle v_{5}^{7}\right\rangle\right\rangle+\left\langle\left\langle v_{8}^{10}\right\rangle\right\rangle\left\langle\left\langle v_{5}^{8}\right\rangle\right\rangle^{2}\left(\omega / \Gamma^{e / i}\right)^{2}}{\Theta \Delta}-\frac{5}{2}, \\
\beta_{\wedge}=\frac{\left.\omega\left\langle\left\langle v_{5}^{8}\right\rangle\right\rangle\left[\left\langle v_{8}^{10}\right\rangle\right\rangle-\left\langle\left\langle v_{5}^{7}\right\rangle\right\rangle\right]}{\Gamma^{e / i} \Theta \Delta}, \\
+\frac{4 \pi \Gamma^{e / i}}{3 \Theta^{2}}\left\{\left\langle v^{9}\right\rangle-\left\langle v^{7}\right\rangle \frac{\left\langle\left\langle v_{5}^{7}\right\rangle\right\rangle+\left\langle\left\langle v_{5}^{10}\right\rangle\right\rangle\left\langle\left\langle u_{5}^{8}\right\rangle\right\rangle^{2}\left(\omega / \Gamma^{e / i}\right)^{2}}{\Delta}\right. \\
\left.+\left\langle v^{5}\right\rangle \frac{\left.\left\langle\left\langle v_{5}^{8}\right\rangle\right\rangle\left\langle\left\langle v_{5}^{10}\right\rangle\right\rangle\left(\omega / \Gamma^{e / i}\right)^{2}\left[\left\langle v_{8}^{10}\right\rangle\right\rangle-\left\langle\left\langle v_{5}^{7}\right\rangle\right\rangle\right]}{\Delta}\right\} \\
\kappa_{\wedge}=\frac{4 \pi \omega}{3 \Theta^{2}}\left\{\left\langle v^{12}\right\rangle+\left\langle v^{8}\right\rangle \frac{\left\langle\left\langle v_{5}^{7}\right\rangle\right\rangle^{2}-\left\langle\left\langle v_{5}^{10}\right\rangle\right\rangle^{2}\left(\omega / \Gamma^{e / i}\right)^{2}}{\Delta}\right. \\
\left.-2\left\langle v^{5}\right\rangle \frac{\left.\left\langle v_{5}^{10}\right\rangle\right\rangle\left\langle\left\langle v_{5}^{7}\right\rangle\right\rangle}{\Delta}\right\},
\end{gathered}
$$

where

$$
\left\langle v^{i}\right\rangle=\int_{0}^{\infty} \frac{f_{M}}{v^{6} \omega^{2}+\left(\Gamma^{e / i}\right)^{2}} v^{i+2} d v,
$$

with the Maxwell-Boltzmann distribution, $f_{M}(v)=$ $n \exp \left(-m v^{2} / 2 k_{B} T\right) /\left(2 \pi m k_{B} T\right)^{1 / 2}, \quad\left\langle\left\langle v_{i^{\prime}}^{i}\right\rangle\right\rangle \equiv\left\langle v^{i}\right\rangle /\left\langle v^{i^{\prime}}\right\rangle$ 

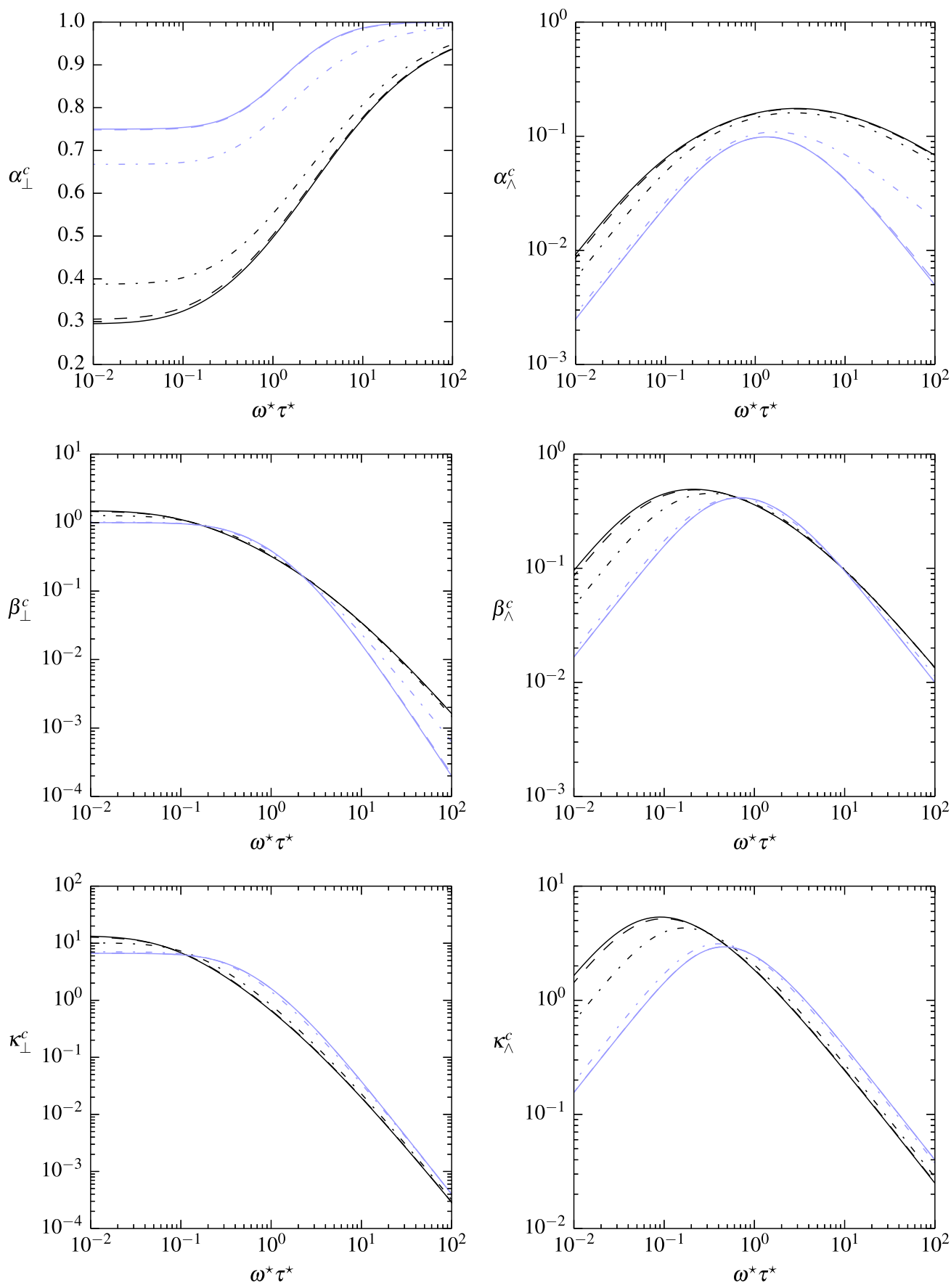

$$
-\Theta=0 \quad-\Theta=0.01 \quad \cdots \quad \Theta=0.1 \quad \cdots \quad \Theta=1 \quad \cdots \quad \Theta=10 \quad \Theta=\infty
$$

FIG. 2. The relativistic transport coefficients as a function of $\omega^{\star} \tau^{\star}$ in the case of a Lorentzian plasma for $\Theta=0$ (black, solid), $\Theta=0.01$ (black, dash), $\Theta=0.1$ (black, dot-dash), $\Theta=1$ (blue, dot-dash), $\Theta=10$ (blue, dash), and $\Theta=\infty$ (blue, solid).

and

find

$$
\lim _{\Theta \rightarrow 0} \Delta=1+\left(\omega\left\langle\left\langle v_{5}^{8}\right\rangle\right\rangle / \Gamma^{e / i}\right)^{2}
$$

The second limit of interest is that which characterizes unmagnetized plasmas: $\omega \rightarrow 0$. In this case we

$$
\begin{aligned}
\lim _{\omega \rightarrow 0}\left\langle u^{i, j}\right\rangle & =\frac{1}{\left(\Gamma^{e / i}\right)^{2}} \int_{0}^{\infty} f_{J} u^{i+2} \gamma^{j-4} d u, \\
\lim _{\omega \rightarrow 0} \Delta & =1
\end{aligned}
$$


and, using

$$
\left\langle u^{5, j}\right\rangle=\frac{n \xi_{j}(\Theta) c^{5}}{4 \pi\left(\Gamma^{e / i}\right)^{2} \Theta^{2-j} K_{2}(1 / \Theta)},
$$

where

$$
\begin{aligned}
\xi_{1}(\Theta)= & E_{1}(1 / \Theta) / \Theta-e^{-1 / \Theta}\left(1-\Theta+2 \Theta^{2}\right. \\
& \left.-6 \Theta^{3}-24 \Theta^{4}-24 \Theta^{5}\right), \\
\xi_{2}(\Theta)= & -E_{1}(1 / \Theta) / \Theta+e^{-1 / \Theta}\left(1-\Theta+2 \Theta^{2}\right. \\
& \left.+42 \Theta^{3}+120 \Theta^{4}+120 \Theta^{5}\right), \\
\xi_{3}(\Theta)= & 48 \Theta^{2} e^{-1 / \Theta}\left(1+6 \Theta+15 \Theta^{2}+15 \Theta^{3}\right),
\end{aligned}
$$

and

$$
E_{1}(x)=\int_{x}^{\infty} \frac{e^{-u} d u}{u}
$$

is the exponential integral function, we can write

$$
\begin{gathered}
\alpha_{\|}=\frac{3 n m_{e} \Gamma^{e / i} \Theta^{2} K_{2}(1 / \Theta)}{\xi_{1}(\Theta) c^{3}}, \\
\beta_{\|}=\frac{\xi_{2}(\Theta)}{\xi_{1}(\Theta)}-\left[\frac{K_{1}(1 / \Theta)}{\Theta K_{2}(1 / \Theta)}+4\right], \\
\kappa_{\|}=\frac{n c^{5}}{3 \Gamma^{e / i} \Theta K_{2}(1 / \Theta)}\left[\xi_{3}(\Theta)-\frac{\left[\xi_{2}(\Theta)\right]^{2}}{\xi_{1}(\Theta)}\right] .
\end{gathered}
$$

It is simple to check that this value of $\alpha_{\|}$agrees with that previously obtained by Braams and Karney [20], and the value of $\kappa_{\|}$similarly agrees with that of Honda and Mima [23].

\section{B. Dimensionless transport coefficients}

By defining a relativistic mean gyro-frequency:

$$
\omega^{\star}=\frac{e B}{\langle\gamma\rangle m_{e}}
$$

and, similarly, a relativistic mean electron-ion collision time [14]:

$$
\tau^{\star}=\frac{3 c^{3}\langle\gamma\rangle \Theta e^{1 / \Theta} K_{2}(1 / \Theta)}{\Gamma^{e / i}\left(1+2 \Theta+2 \Theta^{2}\right)}
$$

[where $\langle\gamma\rangle=3 \Theta+K_{1}(1 / \Theta) / K_{2}(1 / \Theta)$ is the mean energy per particle in a relativistic Maxwellian distribution], it is possible to cast these coefficients into dimensionless form (denoted by the superscript $c$ ), such that they are functions of the atomic number $Z$, Hall parameter $\chi \quad(=$ $\left.\omega^{\star} \tau^{\star}\right)$, and reduced temperature $\Theta$ only, using the following relations:

$$
\begin{aligned}
& \boldsymbol{\alpha}^{c}=\boldsymbol{\alpha}\left(\tau^{\star} /\langle\gamma\rangle m_{e} n\right), \\
& \boldsymbol{\beta}^{c}=\boldsymbol{\beta}, \\
& \boldsymbol{\kappa}^{c}=\boldsymbol{\kappa}\left(\langle\gamma\rangle m_{e} / n k_{B}^{2} T \tau^{\star}\right) .
\end{aligned}
$$

The dimensionless transport coefficients for a Lorentzian plasma are plotted in Fig. 2 as functions of $\omega^{\star} \tau^{\star}$, for six values of $\Theta$ between the classical $(\Theta=0)$ and ultrarelativistic $(\Theta=\infty)$ limits.

The way in which we have defined the Hall parameter, that is, to use the relativistic electron gyro-frequency and mean electron-ion collision time, rather than Braginskii's definitions [15], means that the dimensionless coefficients are independent of temperature in both limits. (Other definitions lead to nonfinite values for the coefficients in the limit $\Theta \rightarrow \infty$.) We stress that, by parameterizing the coefficients in this way, we have effectively split the relativistic correction into two variables: the Hall parameter $\chi$ and the reduced temperature $\Theta$. The reason for this is that, in Sec. IV, we shall find that it is significantly easier to provide fits for these functions than for other parametrizations. It does mean, however, that Fig. 2 (and later Fig. 3) does not on its own illustrate the size of the relativistic correction at different temperatures. For this reason, in Sec. V we shall examine the magnitude of the correction, such that it is clear when it is necessary to use these results in favor of those of classical transport theory.

\section{PLASMAS WITH ARBITRARY ATOMIC NUMBER: NUMERICAL SOLUTION}

In this section, we calculate the transport coefficients for a relativistic plasma under the influence of arbitrary electromagnetic fields, including the effects of both electron-electron and electron-ion scattering. Our approach is analogous to that taken in Sec. II, although the analytical work is dropped in favor of the direct numerical solution of the linearized Boltzmann equation.

As our previous results in Ref. [10] are expressed in spherical coordinates, we shall switch to the spherical harmonic form of the expansion for the distribution function and collision operator, e.g.,

$$
f(\mathbf{r}, \mathbf{u}, t)=\sum_{l=0}^{\infty} \sum_{m=-l}^{l} f_{l}^{m}(\mathbf{r}, u, t) P_{l}^{|m|}(\cos \theta) e^{i m \phi},
$$

where $f_{l}^{-m}=\left(f_{l}^{m}\right)^{*}$. This is formally equivalent to the Cartesian tensor expansion used in the previous section [30]. Without loss of generality, the driving force $\mathbf{s} \in\{\mathbf{E}, \nabla T\}$ can be specified to be in the $x$ direction, with the magnetic field $\mathbf{B}$ in the $z$ direction, as before. The $\mathbf{f}_{1}$ equation [Eq. (10)] is then given in spherical coordinates by [31]

$$
\begin{aligned}
\frac{\partial f_{1}^{0}}{\partial t}= & -v \frac{\partial f_{0}^{0}}{\partial x}+\frac{e E_{x}}{m_{e}} \frac{\partial f_{0}^{0}}{\partial u}-\frac{2 e B_{z}}{\gamma m_{e}} f_{1}^{1} \\
& +C^{e / e}\left(f_{1}^{0}\right)+C^{e / i}\left(f_{1}^{0}\right), \\
\frac{\partial f_{1}^{1}}{\partial t}= & \frac{e B_{z}}{2 \gamma m_{e}} f_{1}^{0}+C^{e / e}\left(f_{1}^{1}\right)+C^{e / i}\left(f_{1}^{1}\right),
\end{aligned}
$$



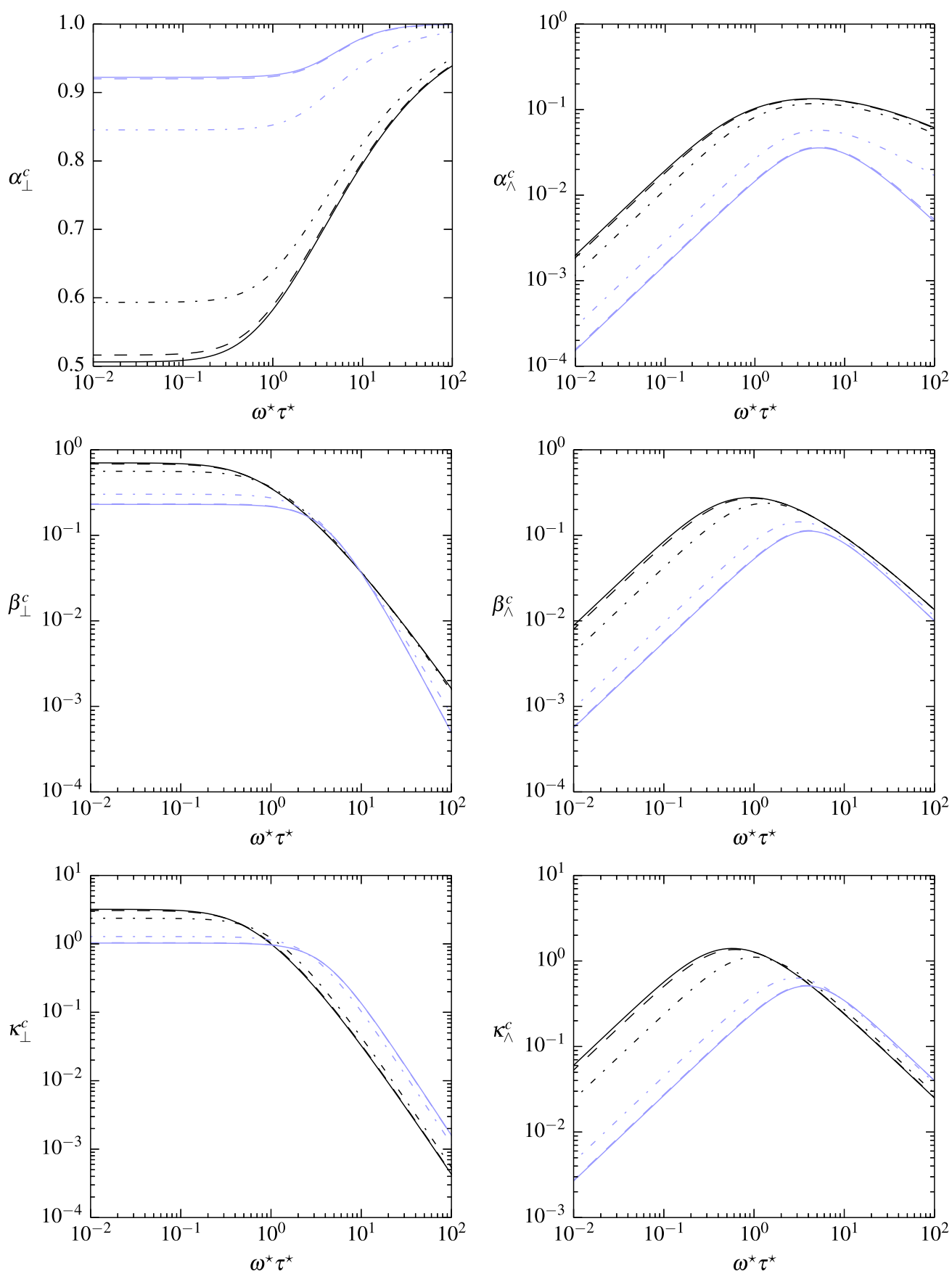

$$
-\Theta=0--\Theta=0.01-\Theta \quad \Theta=0.1 \quad \cdots \quad \Theta=1 \quad-\quad \Theta=10 \quad-\quad \Theta=\infty
$$

FIG. 3. The relativistic transport coefficients as a function of $\omega^{\star} \tau^{\star}$ in the case of a $Z=1$ plasma for $\Theta=0$ (black, solid), $\Theta=0.01$ (black, dash), $\Theta=0.1$ (black, dot-dash), $\Theta=1$ (blue, dot-dash), $\Theta=10$ (blue, dash), and $\Theta=\infty$ (blue, solid).

where, in this case, $\Im\left\{f_{1}^{1}\right\}=0$, such that $f_{1}^{1}=f_{1}^{-1}$. (Note that, in contrast to the previous section, we now retain the time-derivative terms $\partial f_{1}^{m} / \partial t$.) For plasmas with finite atomic number, electron-electron collisions must also be accounted for in the collision operator. This term is given by $[20,32]$

$$
C^{e / e}\left(f_{1}^{m}\right)=\frac{C^{e / e}\left[f_{1}^{m} P_{1}^{|m|}(\cos \theta) e^{i m \phi}, f_{J}\right]+C^{e / e}\left[f_{J}, f_{1}^{m} P_{1}^{|m|}(\cos \theta) e^{i m \phi}\right]}{P_{1}^{|m|}(\cos \theta) e^{i m \phi}},
$$


where

$$
\frac{C^{e / e}\left[f_{1}^{m} P_{1}^{|m|}(\cos \theta) e^{i m \phi}, f_{J}\right]}{P_{1}^{|m|}(\cos \theta) e^{i m \phi}}=-\frac{1}{u^{2}} \frac{\partial}{\partial u}\left(u^{2} F_{u, 0}^{e / e} f_{1}^{m}-u^{2} D_{u u, 0}^{e / e} \frac{\partial f_{1}^{m}}{\partial u}\right)-\frac{2}{u^{2}} D_{\theta \theta, 0}^{e / e} f_{1}^{m},
$$

with $\mathbf{F}^{e / e}$ and $\mathrm{D}^{e / e}$ given by Eqs. (16) to (18) in Ref. [10]. The second term is [20]

$$
\begin{aligned}
\frac{C^{e / e}\left[f_{J}, f_{1}^{m} P_{1}^{|m|}(\cos \theta) e^{i m \phi}\right]}{P_{1}^{|m|}(\cos \theta) e^{i m \phi}}= & \frac{4}{n}\left\{\frac{\Gamma^{e / e}}{\gamma} f_{1}^{m}(u)+\int_{0}^{u}\left[\frac{1}{u^{2} c^{2}}\left(2 j_{1[1] 1}^{\prime}+\frac{j_{1[1] 2}^{\prime}}{\Theta}-10 \frac{j_{1[2] 02}^{\prime}}{\Theta_{e}}\right)+\frac{\gamma}{u^{2} c^{2}}\right.\right. \\
& \left.\times\left(-2 \frac{j_{1[1] 1}^{\prime}}{\Theta}+4 \frac{j_{1[2] 11}^{\prime}}{\Theta}+6 \frac{j_{1[2] 02}^{\prime}}{\Theta^{2}}-24 \frac{j_{1[3] 022}^{\prime}}{\Theta^{2}}\right)+\left(\frac{j_{1[1] 0}^{\prime}}{c^{4} \Theta}\right)+\gamma\left(2 \frac{j_{1[2] 02}^{\prime}}{c^{4} \Theta^{2}}\right)\right] \frac{c u^{\prime 2}}{\gamma \gamma^{\prime}} f_{1}^{m}\left(u^{\prime}\right) d u^{\prime} \\
& +\int_{u}^{\infty}\left[\frac{1}{u^{\prime 2} c^{2}}\left(2 j_{1[1] 1}+\frac{j_{1[1] 2}}{\Theta}-10 \frac{j_{1[2] 02}}{\Theta}\right)+\frac{\gamma^{\prime}}{u^{\prime 2} c^{2}}\left(-2 \frac{j_{1[1] 1}}{\Theta}+4 \frac{j_{1[2] 11}}{\Theta}\right.\right. \\
& \left.\left.\left.+6 \frac{j_{1[2] 02}}{\Theta^{2}}-24 \frac{j_{1[3] 022}}{\Theta^{2}}\right)+\left(\frac{j_{1[1] 0}}{c^{4} \Theta}\right)+\gamma^{\prime}\left(2 \frac{j_{1[2] 02}}{c^{4} \Theta^{2}}\right)\right] \frac{c u^{\prime 2}}{\gamma \gamma^{\prime}} f_{1}^{m}\left(u^{\prime}\right) d u^{\prime}\right\} .
\end{aligned}
$$

where $\quad \Gamma^{e / e}=n e^{4} \ln \Lambda^{e / e} /\left(4 \pi \epsilon_{0}^{2} m_{e}^{2}\right), \quad \ln \Lambda^{e / e} \quad$ is $\quad$ the electron-electron Coulomb logarithm, $j_{l[k] *}=j_{l[k] *}(u / c)$ and $j_{l[k] *}^{\prime}=j_{l[k] *}\left(u^{\prime} / c\right)$; these functions are cataloged for reference in Appendix. In using the form for the collision operator given by Eq. (42), we have neglected the self-interaction of the perturbation, i.e., assumed $\sum_{k} C^{e / e} \quad\left[f_{1}^{m} P_{1}^{|m|}(\cos \theta) e^{i m \phi}, f_{1}^{k} P_{1}^{|k|}(\cos \theta) e^{i k \phi}\right]=0$, and made use of the fact that $C^{e / e}\left[f_{J}, f_{J}\right]=0$ (the collision operator vanishes in equilibrium). As we have neglected all terms $l>1$ in the expansion of the distribution function, we also make the equivalent approximation in the expansion of the collision operator.

Finally, the electron-ion collision term is given by

$$
C^{e / i}\left(f_{1}^{m}\right)=-v_{e i} f_{1}^{m}
$$

cf. Eq. (8).

\section{A. Numerical scheme}

As in classical transport theory, outside the Lorentz limit, one must calculate the transport coefficients numerically. We again need to find the steady-state solution $f_{1}^{0}, f_{1}^{1}(u, t \rightarrow \infty)$, as this allows us to determine the electric current $\mathbf{j}$ and heat flow q using Eqs. (14) and (15), as well as the conversion between notations,

$$
\mathbf{f}_{1}=\left(\begin{array}{c}
f_{1}^{0} \\
2 \Re\left(f_{1}^{1}\right) \\
-2 \Im\left(f_{1}^{1}\right)
\end{array}\right),
$$

where $\Re(z)$ and $\Im(z)$ refer to the real and imaginary parts of $z$, respectively. From this we are then able to calculate the transport coefficients as before.
In order to calculate $f_{1}^{0}, f_{1}^{1}(u, t \rightarrow \infty)$, we transform the differential equations [Eqs. (41)] into algebraic equations using the finite difference method. The momentum of the system is discretized on a uniform grid whose spacing $\Delta u=$ $u_{\max } / N_{u}$, where $u_{\max }$ is the maximum momentum considered and $N_{u}$ the number of computational grid points used. We take $N_{u}=1000$, and the maximum momentum is chosen such that $f_{J}\left(u_{\max }\right)=f_{J}(0) \times 10^{-9}$; the contribution from momenta above this is assumed to be negligible. (This can be checked by varying $u_{\max }$.) The $j$ th momentum point is given by $u_{j}=j \Delta u$

Similarly, time is quantized into discrete steps $\Delta t=$ $t_{\max } / N_{t}$, where, in this case, the number of time steps $N_{t}$ and therefore the total simulation time $t_{\max }$ are both adapted in the simulation; ultimately they are determined by the time $t_{\max }=N_{t} \Delta t$ taken for a steady state to be reached.

The numerical scheme we use in this work is similar to that which has been applied previously by Braams and Karney [20] and Spitzer and Härm [25] to the calculation of transport coefficients. The magnetic field terms in Eqs. (41) and differential terms of the collision operator, Eqs. (43) and (45), are all differenced fully implicitly. The integral term [Eq. (44)] is treated explicitly: this is justified as this term represents the stopping of the bulk of the distribution $f_{J}$ on the perturbation $f_{1}$, an effect that is expected to be small (given the condition on the validity of linear transport theory that the system is close to equilibrium; see Sec. VI). The distribution is then advanced in time using Euler differencing until a steady state is reached. This is considered to have been achieved once the fractional difference in the perturbation $f_{1}^{m}$ between successive time steps is less than $1 \times 10^{-9}$. 
On substituting this representation into Eqs. (41), we arrive at the following band-diagonal series of equations:

$$
\left(\begin{array}{ccccccc}
1+c_{1} \Delta t & d_{1} \Delta t & e_{1} \Delta t & & & \\
b_{1} \Delta t & 1+c_{1} \Delta t & d_{1} \Delta t & e_{1} \Delta t & & \\
a_{2} \Delta t & b_{2} \Delta t & 1+c_{2} \Delta t & d_{2} \Delta t & e_{2} \Delta t & & \\
& a_{2} \Delta t & b_{2} \Delta t & 1+c_{2} \Delta t & d_{2} \Delta t & e_{2} \Delta t & \\
& & a_{3} \Delta t & b_{3} \Delta t & \ldots & \ldots & \ldots \\
& & & a_{3} \Delta t & \ldots & \ldots & \ldots
\end{array}\right) \cdot\left(\begin{array}{c}
f_{1,1}^{0, n+1} \\
f_{1,1}^{1, n+1} \\
f_{1,2}^{0 n+1} \\
f_{1,2}^{1, n+1} \\
\cdots \\
\cdots \\
\end{array}\right.
$$

where

$$
\begin{gathered}
a_{j}=-\frac{D_{u u, j}}{(\Delta u)^{2}}+\frac{1}{2 \Delta u}\left(\frac{2 D_{u u, j}}{u_{j}}+\frac{\partial D_{u u, j}}{\partial u}+F_{u, j}\right), \quad b_{j}=-\frac{e B_{z}}{2 \gamma_{j} m_{e}}, \quad c_{j}=\frac{2 D_{u u, j}}{(\Delta u)^{2}}+\frac{2 D_{\theta \theta, j}}{u_{j}^{2}}+\frac{\Gamma^{e / i}}{u_{j}^{2} v_{j}}, \\
d_{j}=\frac{2 e B_{z}}{\gamma_{j} m_{e}}, \quad e_{j}=-\frac{D_{u u, j}}{(\Delta u)^{2}}-\frac{1}{2 \Delta u}\left(\frac{2 D_{u u, j}}{u_{j}}+\frac{\partial D_{u u, j}}{\partial u}+F_{u, j}\right), \\
A_{j}^{n}=\frac{C^{e / e}\left[f_{J}, f_{1, j}^{0, n} P_{1}^{|m|}(\cos \theta) e^{i m \phi}\right]}{P_{1}^{|m|}(\cos \theta) e^{i m \phi}}+\frac{v_{j}}{T} \frac{\partial T}{\partial x}\left[\frac{\gamma_{j}}{\Theta}-\frac{K_{1}(1 / \Theta)}{\Theta K_{2}(1 / \Theta)}-4\right]+\frac{e E_{x} v_{j}}{k_{B} T}, \quad B_{j}^{n}=\frac{C^{e / e}\left[f_{J}, f_{1, j}^{1, n} P_{1}^{|m|}(\cos \theta) e^{i m \phi}\right]}{P_{1}^{|m|}(\cos \theta) e^{i m \phi}}
\end{gathered}
$$

with $\gamma_{j}=\left(1+u_{j}^{2} / c^{2}\right)^{1 / 2}$ and $v_{j}=u_{j} / \gamma_{j}$

Finally, we are required to specify the initial and boundary conditions of the system: for these we take $f_{1}^{0}, f_{1}^{1}(u, t=0)=0$ (though this can be chosen arbitrarily), and $f_{1}^{0}, f_{1}^{1}(u=0, t)$ and $f_{1}^{0}, f_{1}^{1}\left(u=u_{\text {max }}, t\right)=0$ are enforced throughout the simulation. The implicit differencing scheme allows a steady state to be reached in $\mathcal{O}(1)$ time steps. However, it has been verified that the the same answers are obtained for a time step that is a small fraction of the relevant timescale: the mean electron-ion collision time $\tau^{\star}$ for weakly magnetized plasmas and the reciprocal of the gyro-frequency $1 / \omega^{\star}$ for strongly magnetized plasmas.

We note that this approach is markedly different to that of the previous section, due to the retention of the term $\partial f_{1}^{m} / \partial t$, which enables us to model the evolution of a plasma with time. However, as we are interested in steady-state quantities, this is not strictly necessary; for example, Epperlein and Haines have successfully determined the transport coefficients of a classical plasma while neglecting the time derivative of the perturbation [33].

Once the steady-state solutions of Eqs. (41) have been found, the transport coefficients can be calculated by an integration over momentum space and the use of the relativistic Braginskii transport relations (analogous to the previous section). As an example, the dimensionless transport coefficients for a $Z=1$ plasma are plotted in Fig. 3 in a similar form to those for a Lorentzian plasma.

As a means of benchmarking these numerical results, we have verified that they are consistent with others in three separate limits. First, in the limit $\Theta \rightarrow 0$, the transport coefficients of both Spitzer [34] and Braginskii [15] can be reproduced for all values of $Z$. Second, in the $\omega^{\star} \tau^{\star} \rightarrow 0$ limit, the electrical conductivity $\alpha_{\|}^{c}$ is consistent with the results of Braams and Karney [20], again for arbitrary Z. Finally, in the $Z \rightarrow \infty$ limit, the transport coefficients reduce to those derived in the previous section.

\section{RATIONAL FITS TO THE TRANSPORT COEFFICIENTS}

To enable the coefficients derived in this work to be used in transport calculations, rational functions have been fitted to the numerical data. These take the general form

$$
\varphi_{\perp, \wedge}^{c}=\frac{g(\chi)+\Theta(1+\Theta) h(\chi)}{G(\chi)+\Theta(1+\Theta) H(\chi, \Theta)},
$$

where $\chi=\omega^{\star} \tau^{\star}, g(\chi), G(\chi)$, and $h(\chi)$ are polynomials and $H=H(\chi)$, other than in the case of $\alpha_{\wedge}^{c}$ and $\beta_{\perp}^{c}$, for which $H=H(\chi, \theta)$. In the classical limit, the fits reduce to

$$
\lim _{\Theta \rightarrow 0} \varphi_{\perp, \wedge}^{c}=\frac{g(\chi)}{G(\chi)}
$$

and in the ultrarelativistic limit,

$$
\lim _{\Theta \rightarrow \infty} \varphi_{\perp, \wedge}^{c}=\frac{h(\chi)}{H(\chi)} .
$$

The parameters of the functions $g(\chi), G(\chi), h(\chi)$, and $H(\chi, \Theta)$ are dependent only on the atomic number $Z$. These have been optimized via the use of a nonlinear least squares method, such that the fits accurately reproduce numerically calculated values for each coefficient. In our analysis, the numerical data were spaced at equal logarithmic intervals in the range $10^{-3} \leqslant \omega^{\star} \tau^{\star} \leqslant 10^{3}$ and $10^{-4} \leqslant \Theta \leqslant 10^{2}$. The fits are constructed in such a way, by constraining various parameters, that they reproduce the numerical data exactly in the four limits $(\chi, \Theta) \rightarrow(0,0),(0, \infty),(\infty, 0),(\infty, \infty)$. The full forms of the transport coefficients are given by

$$
\alpha_{\perp}^{c}=1-\frac{a_{1}+a_{2} \chi+\Theta(1+\Theta) a_{3}}{\left(a_{4}+a_{5} \chi+\chi^{2}\right)+\Theta(1+\Theta)\left(a_{6}+a_{7} \chi+\chi^{2}\right)},
$$


TABLE I. The constant coefficients of the rational functions of $\chi$ and $\Theta$ used to fit $\alpha_{\perp}^{c}$ and $\alpha_{\wedge}^{c}$, as per Eqs. (51) and (52), respectively.

\begin{tabular}{|c|c|c|c|c|c|c|c|c|c|c|c|c|c|c|c|}
\hline Z & 1 & 2 & 3 & 4 & 5 & 6 & 7 & 8 & 10 & 12 & 14 & 20 & 30 & 60 & $\infty$ \\
\hline$a_{1}$ & 316 & 159 & 112 & 89.5 & 76.6 & 68.2 & 62.3 & 58.0 & 51.9 & 48.0 & 45.2 & 40.1 & 36.3 & 32.4 & 28.5 \\
\hline$a_{2}$ & 12.0 & 10.2 & 9.36 & 8.88 & 8.57 & 8.35 & 8.18 & 8.04 & 7.85 & 7.71 & 7.61 & 7.42 & 7.25 & 7.08 & 6.89 \\
\hline$a_{3}$ & 109 & 66.3 & 51.7 & 44.3 & 39.9 & 36.9 & 34.7 & 33.0 & 30.7 & 29.2 & 28.0 & 26.0 & 24.4 & 22.7 & 21.0 \\
\hline$a_{4}$ & 639 & 279 & 185 & 143 & 120 & 105 & 95.2 & 87.8 & 77.7 & 71.2 & 66.6 & 58.6 & 52.4 & 46.4 & 40.4 \\
\hline$a_{5}$ & 142 & 86.4 & 67.7 & 58.2 & 52.5 & 48.7 & 45.9 & 43.9 & 40.9 & 38.9 & 37.5 & 34.9 & 32.8 & 30.7 & 28.4 \\
\hline$a_{6}$ & 1390 & 573 & 373 & 287 & 240 & 210 & 190 & 175 & 156 & 143 & 134 & 118 & 107 & 95.3 & 83.9 \\
\hline$a_{7}$ & 229 & 146 & 117 & 102 & 93.3 & 87.3 & 82.9 & 79.6 & 75.0 & 71.9 & 69.7 & 65.6 & 62.4 & 59.1 & 55.6 \\
\hline$A_{1}$ & 17.9 & 16.3 & 12.7 & 10.3 & 8.75 & 7.72 & 7.00 & 6.47 & 5.77 & 5.32 & 5.03 & 4.55 & 4.24 & 4.00 & 3.88 \\
\hline$A_{2}$ & 2.53 & 2.53 & 2.53 & 2.53 & 2.53 & 2.53 & 2.53 & 2.53 & 2.53 & 2.53 & 2.53 & 2.53 & 2.53 & 2.53 & 2.53 \\
\hline$A_{3}$ & 1.5 & 1.5 & 1.5 & 1.5 & 1.5 & 1.5 & 1.5 & 1.5 & 1.5 & 1.5 & 1.5 & 1.5 & 1.5 & 1.5 & 1.5 \\
\hline$A_{4}$ & 6.72 & 8.85 & 8.33 & 7.69 & 7.18 & 6.80 & 6.50 & 6.28 & 5.95 & 5.73 & 5.58 & 5.31 & 5.12 & 4.95 & 4.83 \\
\hline$A_{5}$ & 0.5 & 0.5 & 0.5 & 0.5 & 0.5 & 0.5 & 0.5 & 0.5 & 0.5 & 0.5 & 0.5 & 0.5 & 0.5 & 0.5 & 0.5 \\
\hline$A_{6}$ & 89.8 & 49.8 & 30.5 & 21.5 & 16.6 & 13.6 & 11.7 & 10.3 & 8.63 & 7.61 & 6.94 & 5.87 & 5.17 & 4.59 & 4.16 \\
\hline$A_{7}$ & 86.8 & 77.0 & 60.2 & 49.6 & 42.9 & 38.4 & 35.3 & 33.0 & 30.0 & 28.2 & 26.9 & 25.0 & 23.7 & 22.9 & 22.6 \\
\hline$A_{8}$ & 20.6 & 18.1 & 16.5 & 15.5 & 14.9 & 14.4 & 14.1 & 13.9 & 13.6 & 13.4 & 13.2 & 13.0 & 12.8 & 12.6 & 12.4 \\
\hline$A_{9}$ & 440 & 247 & 154 & 111 & 87.1 & 72.5 & 62.8 & 56.0 & 47.0 & 41.5 & 37.8 & 31.6 & 27.1 & 23.1 & 19.3 \\
\hline
\end{tabular}

$$
\begin{gathered}
\alpha_{\wedge}^{c}=\frac{\chi\left[A_{1}+A_{2} \chi+A_{3} \Theta(1+\Theta)\left(A_{4}+A_{5} \chi\right)\right]}{\left(A_{6}+A_{7} \chi+A_{8} \chi^{2}+\chi^{8 / 3}\right)+A_{3} \Theta(1+\Theta)\left(A_{9}+A_{7} \chi+A_{8} \chi^{2}+\chi^{8 / 3+\Theta / 3(\Theta+1)}\right)}, \\
\beta_{\perp}^{c}=\frac{b_{1}+b_{2} \chi+\Theta(1+\Theta)\left(b_{3}+b_{4} \chi\right)}{\left(b_{5}+b_{6} \chi+b_{7} \chi^{2}+\chi^{8 / 3}\right)+\Theta(1+\Theta)\left(b_{8}+b_{9} \chi+b_{10} \chi^{2}+\chi^{8 / 3+b_{11} \Theta / 3\left(b_{11} \Theta+1\right)}\right)}, \\
\beta_{\wedge}^{c}=\frac{\chi\left[B_{1}+B_{2} \chi+B_{3} \Theta(1+\Theta)\left(B_{4}+\chi\right)\right]}{\left(B_{5}+B_{6} \chi+B_{7} \chi^{2}+\chi^{3}\right)+B_{3} \Theta(1+\Theta)\left(B_{8}+B_{6} \chi+B_{7} \chi^{2}+\chi^{3}\right)}, \\
\kappa_{\perp}^{c}=\frac{c_{1}+c_{2} \chi+c_{3} \Theta(1+\Theta)\left(c_{4}+c_{5} \chi\right)}{\left(c_{6}+c_{7} \chi+c_{8} \chi^{2}+\chi^{5 / 2}+\chi^{3}\right)+c_{3} \Theta(1+\Theta)\left(c_{9}+c_{10} \chi+c_{11} \chi^{2}+\chi^{3}\right)}, \\
\kappa_{\wedge}^{c}=\frac{\chi\left[C_{1}+C_{2} \chi+C_{3} \Theta(1+\Theta)\left(C_{4}+C_{5} \chi\right)\right]}{\left(C_{6}+C_{7} \chi+C_{8} \chi^{2}+\chi^{3}\right)+C_{3} \Theta(1+\Theta)\left(C_{9}+C_{7} \chi+C_{8} \chi^{2}+\chi^{3}\right)},
\end{gathered}
$$

with

$$
\begin{gathered}
\alpha_{\|}^{c}=\lim _{\chi \rightarrow 0} \alpha_{\perp}^{c}=1-\frac{a_{1}+a_{3} \Theta(1+\Theta)}{a_{4}+a_{6} \Theta(1+\Theta)}, \\
\beta_{\|}^{c}=\lim _{\chi \rightarrow 0} \beta_{\perp}^{c}=\frac{b_{1}+b_{3} \Theta(1+\Theta)}{b_{5}+b_{8} \Theta(1+\Theta)}, \\
\kappa_{\|}^{c}=\lim _{\chi \rightarrow 0} \kappa_{\perp}^{c}=\frac{c_{1}+c_{3} c_{5} \Theta(1+\Theta)}{c_{6}+c_{5} c_{9} \Theta(1+\Theta)} .
\end{gathered}
$$

The parameters of these fits are tabulated for a range of values of $Z$ in Tables I to III.

The $\chi$ dependence of all transport coefficients in the weakfield limit $(\chi \rightarrow 0)$ is not a function of temperature. This is also the case for $\alpha_{\perp}^{c}, \beta_{\wedge}^{c}, \kappa_{\perp}^{c}$, and $\kappa_{\wedge}^{c}$ in the strong-field limit $(\chi \rightarrow$ $\infty)$. However, the $\chi$ dependence of $\alpha_{\wedge}^{c}$ and $\beta_{\perp}^{c}$ in the strongfield limit does vary with temperature: at $\Theta=0, \alpha_{\wedge}^{c} \sim \chi^{-2 / 3}$ and $\beta_{\perp}^{c} \sim \chi^{-5 / 3}$, whereas, at $\Theta=\infty, \alpha_{\wedge}^{c} \sim \chi^{-1}$ and $\beta_{\perp}^{c} \sim$ $\chi^{-2}$. (The former limit was found by Epperlein and Haines [33]; the latter may be obtained by following their analysis in the limit $\gamma \rightarrow u / c$.) For these two coefficients, at intermediate temperatures $(\Theta \sim 1)$, errors may increase with $\chi$ in the strong field limit. However, transport is strongly suppressed for such cases and maximum errors of $20 \%$ are observed for values up to $\chi=10^{3}$. This is shown for the example of a Lorentzian plasma in Fig. 4, along with the fractional percentage errors of the other fits provided. Outside these two special cases, the maximum error is approximately $15 \%$ for all temperatures and field strengths.

\section{DISCUSSION OF RESULTS}

As this work has so far been largely mathematical, in this section we provide some physical interpretation of the previous 
TABLE II. The constant coefficients of the rational functions of $\chi$ and $\Theta$ used to fit $\beta_{\perp}^{c}$ and $\beta_{\wedge}^{c}$, as per Eqs. (53) and (54), respectively.

\begin{tabular}{llllllllllllllll}
\hline \hline $\mathrm{Z}$ & 1 & 2 & 3 & 4 & 5 & 6 & 7 & 8 & 10 & 12 & 14 & 20 & 30 & 60 & $\infty$ \\
\hline$b_{1}$ & 2.07 & 0.905 & 0.646 & 0.525 & 0.453 & 0.405 & 0.371 & 0.345 & 0.311 & 0.286 & 0.269 & 0.237 & 0.212 & 0.185 & 0.158 \\
$b_{2}$ & 4.82 & 4.85 & 4.86 & 4.86 & 4.87 & 4.87 & 4.87 & 4.87 & 4.87 & 4.87 & 4.87 & 4.88 & 4.88 & 4.88 & 4.88 \\
$b_{3}$ & 2.39 & 1.35 & 1.13 & 1.04 & 0.979 & 0.943 & 0.920 & 0.904 & 0.887 & 0.877 & 0.871 & 0.868 & 0.874 & 0.894 & 0.943 \\
$b_{4}$ & 5 & 3.5 & 3 & 2.76 & 2.61 & 2.51 & 2.44 & 2.38 & 2.31 & 2.26 & 2.22 & 2.16 & 2.11 & 2.06 & 2 \\
$b_{5}$ & 2.95 & 1 & 0.635 & 0.481 & 0.395 & 0.342 & 0.305 & 0.278 & 0.242 & 0.218 & 0.202 & 0.172 & 0.150 & 0.127 & 0.105 \\
$b_{6}$ & 7.04 & 5.45 & 4.91 & 4.61 & 4.42 & 4.28 & 4.18 & 4.11 & 3.99 & 3.91 & 3.86 & 3.74 & 3.65 & 3.55 & 3.44 \\
$b_{7}$ & 7.93 & 8.70 & 9.27 & 9.65 & 9.93 & 10.2 & 10.3 & 10.5 & 10.7 & 10.8 & 11.0 & 11.2 & 11.4 & 11.7 & 12.0 \\
$b_{8}$ & 10.4 & 3.77 & 2.54 & 2.03 & 1.75 & 1.58 & 1.46 & 1.37 & 1.26 & 1.19 & 1.14 & 1.06 & 1.01 & 0.965 & 0.943 \\
$b_{9}$ & 18.9 & 8.87 & 6.18 & 4.97 & 4.30 & 3.88 & 3.59 & 3.37 & 3.09 & 2.91 & 2.78 & 2.57 & 2.43 & 2.31 & 2.25 \\
$b_{10}$ & 2.14 & 1.92 & 2.03 & 2.14 & 2.23 & 2.31 & 2.38 & 2.44 & 2.54 & 2.62 & 2.68 & 2.81 & 2.94 & 3.08 & 3.25 \\
$b_{11}$ & 2.5 & 1.75 & 1.5 & 1.25 & 1.25 & 1.25 & 1.25 & 1.25 & 1 & 1 & 1 & 1 & 1 & 1 & 1 \\
$B_{1}$ & 4.86 & 2.83 & 2.34 & 2.11 & 1.96 & 1.86 & 2.10 & 2.07 & 2.01 & 1.97 & 1.93 & 1.89 & 2.09 & 2.14 & 2.48 \\
$B_{2}$ & 1.5 & 1.5 & 1.5 & 1.5 & 1.5 & 1.5 & 1.5 & 1.5 & 1.5 & 1.5 & 1.5 & 1.5 & 1.5 & 1.5 & 1.5 \\
$B_{3}$ & 0.5 & 0.5 & 0.5 & 0.5 & 0.5 & 0.5 & 0.75 & 0.75 & 0.75 & 0.75 & 0.75 & 0.75 & 1 & 1 \\
$B_{4}$ & 6.45 & 4.85 & 4.53 & 4.35 & 4.22 & 4.10 & 4.19 & 4.19 & 4.15 & 4.11 & 4.07 & 3.99 & 4.17 & 4.20 & 4.25 \\
$B_{5}$ & 5.51 & 1.56 & 0.907 & 0.653 & 0.520 & 0.438 & 0.453 & 0.416 & 0.362 & 0.328 & 0.304 & 0.265 & 0.266 & 0.245 & 0.252 \\
$B_{6}$ & 6.15 & 3.21 & 2.57 & 2.28 & 2.10 & 1.99 & 2.21 & 2.18 & 2.13 & 2.09 & 2.07 & 2.05 & 2.26 & 2.37 & 2.81 \\
$B_{7}$ & 9.81 & 7.46 & 6.87 & 6.57 & 6.38 & 6.24 & 6.51 & 6.48 & 6.42 & 6.36 & 6.32 & 6.26 & 6.55 & 6.60 & 7.06 \\
$B_{8}$ & 115 & 33.7 & 19.7 & 14.0 & 11.0 & 9.17 & 8.28 & 7.49 & 6.36 & 5.61 & 5.09 & 4.19 & 3.75 & 3.14 & 2.73 \\
\hline \hline
\end{tabular}

results. Of initial note is that transport in weakly magnetized plasmas $(\chi \ll 1)$ is dominated by collisions, whereas in strongly magnetized plasmas $(\chi \gg 1)$ it is dominated by the magnetic field. In the classical theory [15], this can be thought of in terms of the collisional mean free path $\lambda_{e i}=v_{\text {th }} \tau$ and the Larmor radius $r_{L}=v_{\mathrm{th}} / \omega$ (where $v_{\mathrm{th}}$ is the thermal electron speed); whichever is smaller determines the characteristic step size for transport. Here $\tau$ is Braginskii's mean electron-ion collision time [15]:

$$
\tau=\frac{3 \sqrt{\pi} \Theta^{3 / 2} c^{3}}{\sqrt{2} \Gamma^{e / i}},
$$

which should not be mistaken for its relativistic counterpart seen earlier in this work [Eq. (36)].

At $\chi \sim 1$, these two length scales are of the same order, and transport is both collisional and magnetized. (This is why the $\wedge$ coefficients are maximized at this point [15].) We will see that a similar physical picture applies in the relativistic case.

First, note that we have parameterized the coefficients in terms of $\omega^{\star} \tau^{\star}$, which is the product of the relativistic gyro-frequency $\omega^{\star}$ and the relativistically mean electron-ion collision time $\tau^{\star}$. The reason for doing this can be seen by considering $\alpha_{\perp}^{c}$ : electron-ion collisions set up a frictional

TABLE III. The constant coefficients of the rational functions of $\chi$ and $\Theta$ used to fit $\kappa_{\perp}^{c}$ and $\kappa_{\wedge}^{c}$, as per Eqs. (55) and (56), respectively.

\begin{tabular}{lcccccccccccccccccc}
\hline \hline $\mathrm{Z}$ & 1 & 2 & 3 & 4 & 5 & 6 & 7 & 8 & 10 & 12 & 14 & 20 & 30 & 60 \\
\hline$c_{1}$ & 9.89 & 2.37 & 0.973 & 0.774 & 0.686 & 0.769 & 0.837 & 0.702 & 0.539 & 0.445 & 0.386 & 0.293 & 0.281 & 0.213 & 0.178 \\
$c_{2}$ & 4.58 & 3.87 & 3.63 & 3.51 & 3.44 & 3.40 & 3.36 & 3.34 & 3.30 & 3.28 & 3.26 & 3.23 & 3.21 & 3.19 & 3.16 \\
$c_{3}$ & 1.25 & 1.5 & 1.5 & 1.75 & 2 & 2.5 & 3 & 3 & 3 & 3 & 3 & 3 & 3.5 & 3.5 & 4 \\
$c_{4}$ & 12.5 & 2.81 & 1.25 & 0.903 & 0.732 & 0.674 & 0.627 & 0.543 & 0.440 & 0.380 & 0.342 & 0.282 & 0.249 & 0.212 & 0.181 \\
$c_{5}$ & 16 & 10 & 8 & 7 & 6.4 & 6 & 5.71 & 5.5 & 5.2 & 5 & 4.86 & 4.6 & 4.4 & 4.2 & 4 \\
$c_{6}$ & 3.09 & 0.482 & 0.159 & 0.111 & 0.0894 & 0.0934 & 0.0965 & 0.0775 & 0.0557 & 0.0440 & 0.0368 & 0.0261 & 0.0237 & 0.0168 & 0.0131 \\
$c_{7}$ & 2.74 & 1.08 & 0.688 & 0.579 & 0.521 & 0.512 & 0.510 & 0.467 & 0.412 & 0.378 & 0.355 & 0.317 & 0.301 & 0.270 & 0.247 \\
$c_{8}$ & 5.84 & 3.32 & 2.56 & 2.61 & 2.69 & 3.00 & 3.26 & 3.14 & 2.99 & 2.91 & 2.85 & 2.77 & 2.88 & 2.81 & 2.85 \\
$c_{9}$ & 12.2 & 1.64 & 0.565 & 0.345 & 0.249 & 0.210 & 0.182 & 0.149 & 0.111 & 0.0897 & 0.0768 & 0.0575 & 0.0466 & 0.0359 & 0.0272 \\
$c_{10}$ & 12.0 & 4.85 & 3.10 & 2.31 & 1.88 & 1.60 & 1.41 & 1.29 & 1.13 & 1.02 & 0.951 & 0.824 & 0.724 & 0.632 & 0.541 \\
$c_{11}$ & 1.84 & 0.937 & 0.707 & 0.696 & 0.705 & 0.750 & 0.783 & 0.774 & 0.766 & 0.766 & 0.769 & 0.783 & 0.824 & 0.855 & 0.915 \\
$C_{1}$ & 18.0 & 7.71 & 5.88 & 4.99 & 3.82 & 3.50 & 3.29 & 3.13 & 2.92 & 2.80 & 2.73 & 2.07 & 1.76 & 1.66 & 1.65 \\
$C_{2}$ & 2.5 & 2.5 & 2.5 & 2.5 & 2.5 & 2.5 & 2.5 & 2.5 & 2.5 & 2.5 & 2.5 & 2.5 & 2.5 & 2.5 & 2.5 \\
$C_{3}$ & 0.5 & 0.75 & 0.75 & 0.75 & 1 & 1 & 1 & 1 & 1 & 1 & 1 & 1.5 & 2 & 2 \\
$C_{4}$ & 22.0 & 7.30 & 5.97 & 5.34 & 3.28 & 3.11 & 3.01 & 2.94 & 2.87 & 2.85 & 2.86 & 1.60 & 1.11 & 1.16 & 1.33 \\
$C_{5}$ & 4 & 4 & 4 & 4 & 4 & 4 & 4 & 4 & 4 & 4 & 4 & 4 & 4 & 4 \\
$C_{6}$ & 2.99 & 0.492 & 0.230 & 0.143 & 0.0882 & 0.0688 & 0.0568 & 0.0488 & 0.0391 & 0.0335 & 0.0299 & 0.0192 & 0.0142 & 0.0115 & 0.00957 \\
$C_{7}$ & 3.41 & 1.11 & 0.725 & 0.560 & 0.407 & 0.355 & 0.321 & 0.297 & 0.265 & 0.246 & 0.234 & 0.172 & 0.142 & 0.128 & 0.122 \\
$C_{8}$ & 7.97 & 3.78 & 3.03 & 2.67 & 2.14 & 2.01 & 1.92 & 1.85 & 1.77 & 1.72 & 1.69 & 1.36 & 1.21 & 1.17 & 1.17 \\
$C_{9}$ & 82.2 & 9.66 & 4.60 & 2.91 & 1.39 & 1.09 & 0.908 & 0.785 & 0.632 & 0.544 & 0.488 & 0.219 & 0.124 & 0.101 & 0.0857 \\
\hline \hline
\end{tabular}



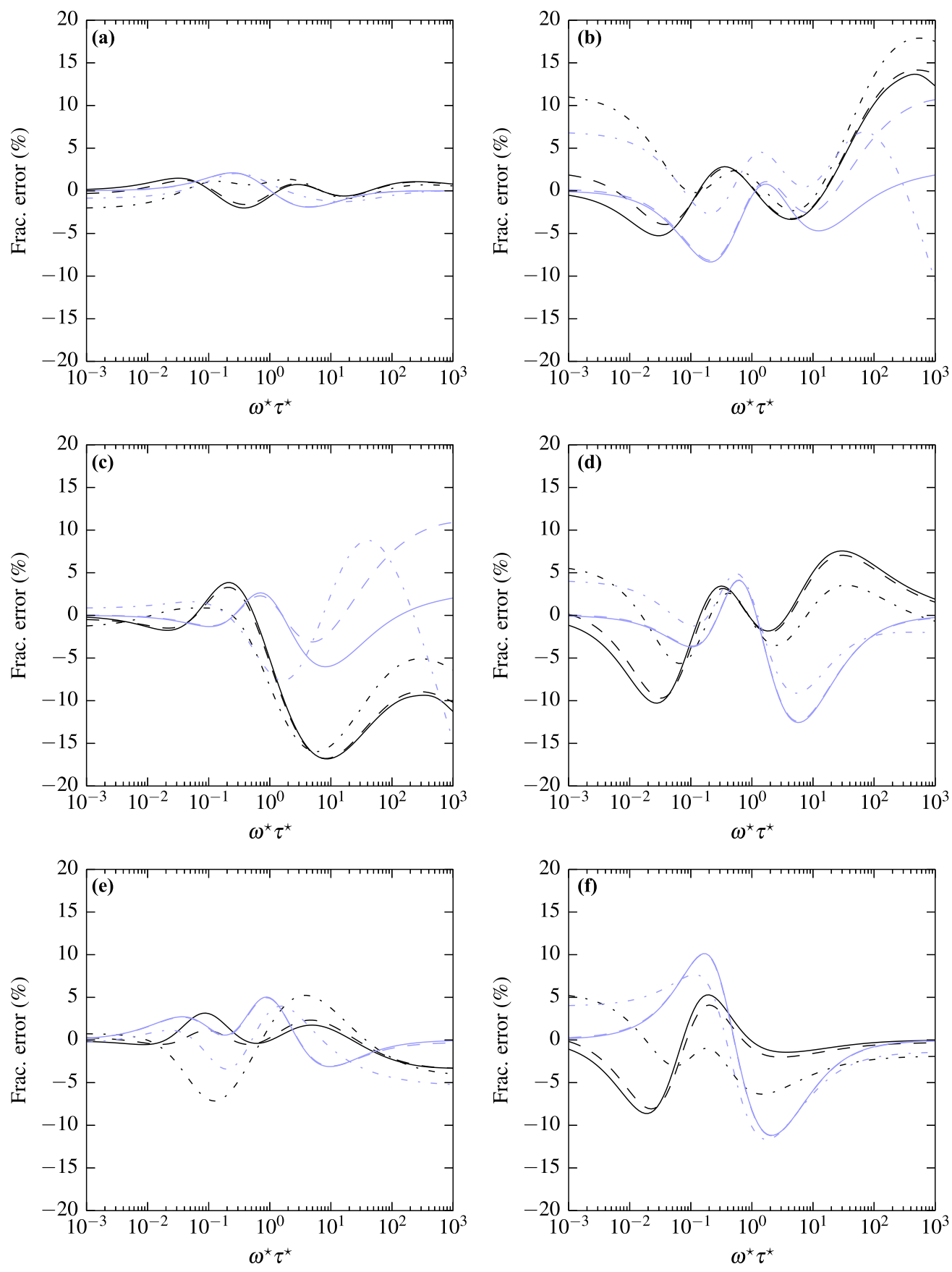

$-\Theta=0 \quad-\Theta=0.01 \quad \cdots \quad \Theta=0.1 \quad \cdots \quad \Theta=1 \quad \cdots \quad \Theta=10 \quad \Theta=\infty$

FIG. 4. Plot of fractional error in fits to (a) $\alpha_{\perp}$, (b) $\alpha_{\wedge}$, (c) $\beta_{\perp}$, (d) $\beta_{\wedge}$, (e) $\kappa_{\perp}$, (f) $\kappa_{\wedge}$ as a function of $\omega^{\star} \tau^{\star}$ in the case of a Lorentzian plasma $(Z \rightarrow \infty)$ for $\Theta=0$ (black, solid), $\Theta=0.01$ (black, dash), $\Theta=0.1$ (black, dot-dash), $\Theta=1$ (blue, dot-dash), $\Theta=10$ (blue, dash), and $\Theta=\infty$ (blue, solid).

force that resists the flow of current, $\mathbf{R}_{e i} \sim \alpha_{\perp}^{c} m_{e} n\langle\gamma\rangle\langle\mathbf{v}\rangle / \tau^{\star}$ (where the current is given by $-e n\langle\mathbf{v}\rangle)$ [14]. The use of $\tau^{\star}$ in our parametrization ensures $\alpha_{\perp}^{c}$ tends to unity in the strong-field limit, corresponding to the case in which the electron distribution is a drifting Maxwellian. For lower values of the Hall parameter, the resistivity $\alpha_{\perp}^{c}$ decreases, because collisions distort the distribution function and the electrons that contribute primarily to the current are those that are less collisional. This difference between the weak- and strong-field resistivity is reduced relativistically (compared to that seen classically) because the electron-ion collision frequency scales with $1 / u^{2} v$ rather than $1 / v^{3}$ [Eq. (9)], with the former tending to $1 / u^{2}$ in the ultrarelativistic case.

This $\omega^{\star} \tau^{\star}$ parametrization also ensures that the functional form of the coefficients is broadly independent of temperature (see, e.g., Fig. 3), and the physical reasoning outlined above 
can be updated as follows: for $\omega^{\star} \tau^{\star} \ll 1$, the collisional mean free path, $\lambda_{e i}=v_{\text {th }} \tau^{\star}$, is the characteristic length scale for transport, and, conversely, for $\omega^{\star} \tau^{\star} \gg 1$, the Larmor radius $r_{L} \sim v_{\text {th }} / \omega^{\star}$ is the appropriate length scale. At $\omega^{\star} \tau^{\star} \sim 1$, these two lengths are of the same order, which is why, for all values of $\Theta$, the switch between collisional and magnetized transport occurs at or near to this point.

We are now in a position to compare these results with those given by the classical theory, so as to analyze the magnitude of relativistic effects at various temperatures and field strengths. For this, it is useful to use the classical form of the Hall parameter, $\psi=\omega \tau$, as this enables us to confine the relativistic correction to the $\Theta$ variable alone. In the classical theory, for a given $\omega \tau$, the dimensionless coefficients are independent of temperature. However, in the relativistic theory, this no longer remains the case. Plotting the ratio of the coefficients (given in terms of $\omega \tau$ ) at arbitrary $\Theta$ to that at $\Theta \approx 0$ therefore provides the size of the relativistic correction. This is done in Fig. 5 for the case of a $Z=1$ plasma. (Corrections for plasmas with different atomic numbers are of the same order.)

Figure 5 shows that the relativistic electrical resistivity $\alpha_{\perp}$ increases from its classical value as the temperature is increased. This can be understood by again considering the frictional force $\mathbf{R}_{e i}$, which balances the electromagnetic fields and pressure gradient in a steady state. As we have seen, $\mathbf{R}_{e i} \sim \alpha_{\perp}^{c} m_{e} n\langle\gamma\rangle\langle\mathbf{v}\rangle / \tau^{\star}$, with $\langle\gamma\rangle / \tau^{\star} \rightarrow 1 / \tau \sim \Theta^{-3 / 2}$ (nonrelativistic) and $\langle\gamma\rangle / \tau^{\star} \sim \Theta^{-1}$ (ultrarelativistic). In the ultrarelativistic limit, the correction to the classical result therefore scales as $\Theta^{1 / 2}$. Physically this represents the increased collisionality of high-temperature plasmas when relativistic effects are accounted for, or, alternatively, the reduction of the current $\mathbf{j}$ and heat flow $\mathbf{q}$ generated in the plasma as particles are limited to $c$.

Similarly, at low field strengths, the thermal conductivity $\kappa_{\perp}$ decreases from its classical value as the temperature is increased. The correction in this case scales as $\Theta^{-1 / 2}$ in the ultrarelativistic limit. This is because the thermal conductivity is effectively a diffusion coefficient of the form $(\Delta x)^{2} / \Delta t$, where $\Delta x$ is the characteristic step length of transport and $\Delta t$ the step time [15]. Classically, this can be expressed $\lambda_{e i}^{2} / \tau=\left(v_{\mathrm{th}} \tau\right)^{2} / \tau$, whose temperature dependence is given by $\left(\Theta^{1 / 2} \Theta^{3 / 2}\right)^{2} / \Theta^{3 / 2} \sim \Theta^{5 / 2}$. In the ultrarelativistic limit, this switches to $\left(v_{\text {th }} \tau^{\star}\right)^{2} / \tau^{\star}$, which scales as $\left(\Theta^{2}\right)^{2} / \Theta^{2} \sim \Theta^{2}$. The corresponding $\Theta^{-1 / 2}$ correction can be attributed to the same physical considerations as the resistivity above.

By contrast, at low field strengths, the thermoelectric coefficient $\beta_{\perp}$ does not change indefinitely at high temperatures. In fact, in the ultrarelativistic limit, $\beta_{\|}^{c} \rightarrow 0.2297$, which is of the same order as the classical value, $\beta_{\|}^{c} \approx 0.7029(Z=1)$. Again this can be justified by simple physical arguments [15]; consider, for example, the thermoelectric term $n k_{B} \boldsymbol{\beta} \cdot \nabla T$ in Eq. (1). This term arises due to the fact that, when a temperature gradient is imposed, the electrons higher up the gradient are less collisional, which produces a net frictional force down the gradient. An estimate for this force can be given by considering an electrons with extra energy $\sim \lambda_{e i} k_{B} \partial T / \partial x$ as they travel a mean free path $\lambda_{e i}$ down the temperature gradient. Thus the force is of order $\left(\lambda_{e i} / T\right) \partial T / \partial x\left(\langle\gamma\rangle m_{e} n v_{\mathrm{th}} / \tau^{\star}\right) \sim n \partial T / \partial x$, which is clearly independent of temperature.
The corrections at high field strengths, and to the $\alpha_{\wedge}$, $\beta_{\wedge}$, and $\kappa_{\wedge}$, coefficients, are slightly more subtle. First, the temperature scaling of $\omega \tau$ (the nonrelativistic Hall parameter) is $\Theta^{3 / 2}$, whereas that of $\omega^{\star} \tau^{\star}$ (the relativistic Hall parameter) is $\Theta$ in the ultrarelativistic limit. In other words, relativistic effects mean it is more difficult to magnetize a plasma than would be expected classically (again because of increased collisionality); the effect of the magnetic field is decreased by $\sim \Theta^{-1 / 2}$ at very high temperatures. With the exception of $\alpha_{\perp}$, this is manifested in an increase in all coefficients in the high field limit. This can be seen as all these decrease rapidly with an increasing magnetic field, and a relativistic treatment acts to reduce the effects of this.

The corrections seen in the low field limit of $\alpha_{\wedge}, \beta_{\wedge}$, and $\kappa_{\wedge}$ are due to the combination of effects discussed above. For $\alpha_{\wedge}$, an $\sim \Theta^{1 / 2}$ increase as per $\alpha_{\perp}$ is exactly balanced by the $\sim \Theta^{-1 / 2}$ decrease due to the reduced magnetization (as the coefficient scales $\sim \chi$ in this limit). Therefore, in the classical limit, $\alpha_{\wedge}^{c}=0.1988 \omega \tau$, whereas in the ultrarelativistic limit, $\alpha_{\wedge}^{c}=0.01528 \omega \tau$. In the case of $\beta_{\perp}$, a simple $\Theta^{-1 / 2}$ correction is required due to magnetization effects. Last, the combination of two $\Theta^{-1 / 2}$ scalings for $\kappa_{\wedge}$ results in a net $\Theta^{-1}$ correction [35].

Finally, we note that the relativistic corrections to $\alpha_{\|}$and $\kappa_{\|}$can be fairly significant, even at mild temperatures. For example, at $k_{B} T=30 \mathrm{keV}(\Theta \approx 0.06), \kappa_{\|}$is reduced to $80 \%$ of its classical value. Note that this is somewhat larger than the correction to $\alpha_{\|}$, as the thermal conductivity is a higher moment of the distribution and thus more sensitive to the change in the shape of the tail due to relativistic effects than the electrical resistivity. However, the greatest corrections are those to the coefficients in the $(\mathbf{b} \times \mathbf{s})$ direction in the weakfield limit. We find that, even at $k_{B} T=5 \mathrm{keV}(\Theta \approx 0.01), \beta_{\wedge}$ and $\kappa_{\wedge}$ are reduced by $10 \%$. By $k_{B} T=20 \mathrm{keV}(\Theta \approx 0.04)$, $\kappa_{\wedge}$ has decreased to approximately $60 \%$ of its classical value. Although transport is strongly suppressed in this direction for weak field strengths, we find these corrections remain to $\omega \tau \sim$ 0.1 ; at this point the thermal conductivity $\kappa_{\wedge}$ is a large fraction of its maximum value (see the $\Theta=0$ case in Fig. 3, for which $\left.\tau=\tau^{\star}\right)$.

\section{LIMITS OF VALIDITY}

First, the results of this work are restricted in their validity to an ideal plasma; that is one which is fully ionized and weakly coupled. The former condition requires the presence of neutral particles to be negligible; otherwise the transport coefficients assume more complex forms [26]. The latter corresponds to $\ln \Lambda^{a / b} \gg 1$, such that small-angle scattering dominates and the Fokker-Planck approach can be accurately used to describe transport [11].

The limits of applicability of linear transport theory have been discussed by numerous authors [36-38]. For a relativistic plasma, it is required that the thermal-averaged momentum,

$$
u_{t h}=\frac{4 \pi}{n} \int f_{J} u^{3} d u=\frac{2\left(1+3 \Theta+3 \Theta^{2}\right)}{e^{1 / \Theta} K_{2}(1 / \Theta)} c
$$



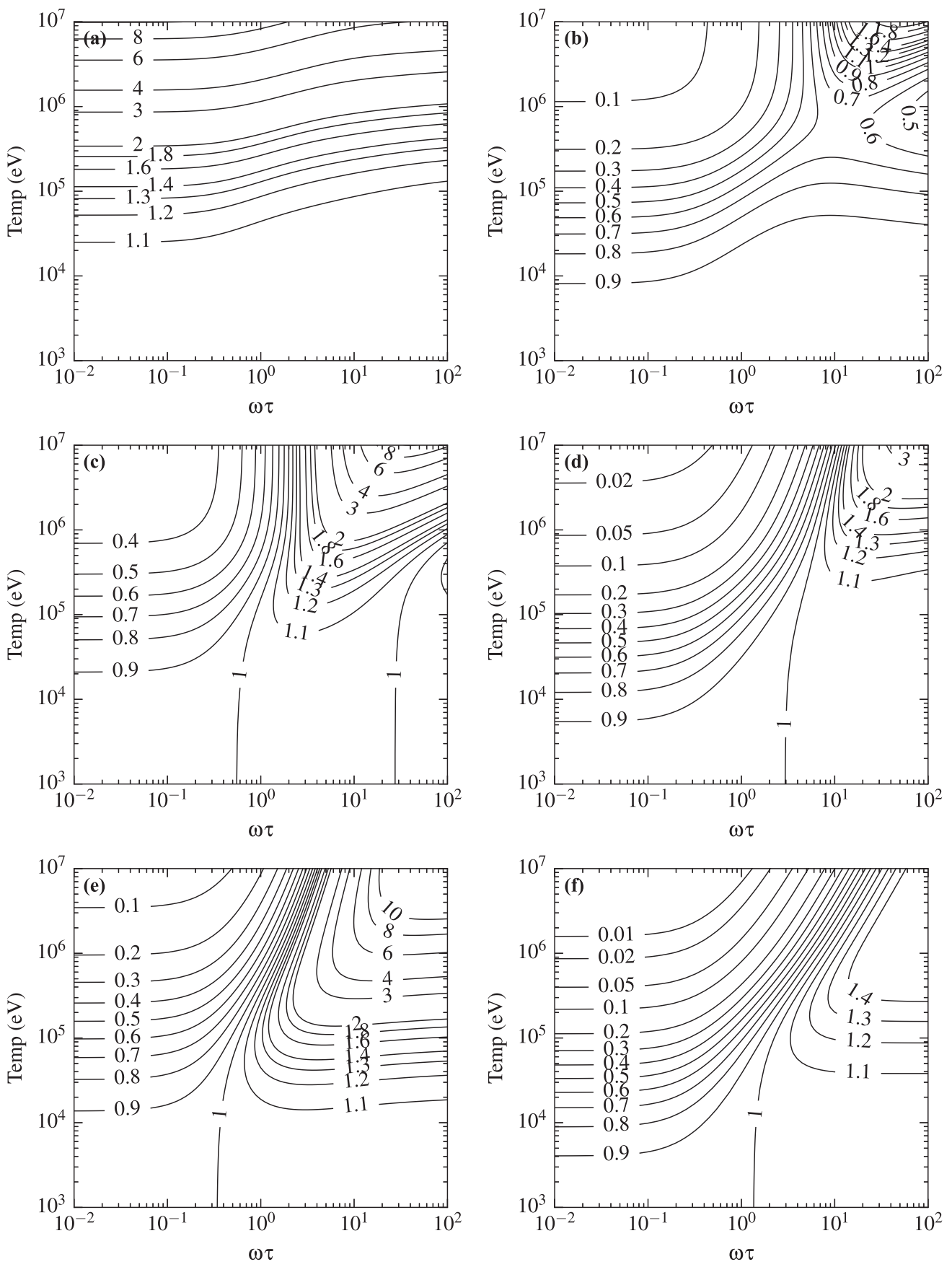

FIG. 5. Relativistic correction factors in the case of (a) $\alpha_{\perp}$, (b) $\alpha_{\wedge}$, (c) $\beta_{\perp}$, (d) $\beta_{\wedge}$, (e) $\kappa_{\perp}$, (f) $\kappa_{\wedge}$ as a function of the nonrelativistic Hall parameter $\psi=\omega \tau$ and reduced temperature $\Theta$ for a $Z=1$ plasma.

is much greater than the magnitude of the drift momentum,

$$
\mathbf{u}_{d r}=\frac{4 \pi}{3 n} \int \mathbf{f}_{1} u^{3} d u
$$

otherwise $\mathbf{f}_{1}$ can no longer be considered to be a small perturbation and the time evolution of $f_{0}, f_{2}$, etc., must be considered. This places constraints on the magnitude of the electric fields $\mathbf{E}$ and temperature gradients $\nabla T$ that may be studied using this approach.

The assumption that the ions are infinitely massive is fairly robust for $T_{i} \sim T_{e}$ and $\Theta_{i} \ll 1$, given $m_{i} \gg m_{e}$. We note that in the presence of a strong magnetic field the ion contribution

to transport may be greater than the electron contribution in the direction normal to the magnetic field [39]. However, the higher mass of the ions means that, for temperatures as high as $k_{B} T_{i} \sim 0.1 \mathrm{GeV}\left(\Theta_{e} \sim 100\right)$, their motion remains nonrelativistic. For this, the reader is referred to earlier works on classical ion transport $[15,26]$.

In the present work, our analysis has been confined to the inertial frame in which the ions are at rest. Allowing for relativistic flow is complicated: Dzhavakhishvili and Tsintsadze [14] showed that the relativistic MHD equations contained terms $\left(\sim 1 / c^{2}\right)$ completely absent from their classical counterparts [15]. However, in the case of a mildly 
relativistic plasma, in which the mean velocity of the ions $\mathbf{V}_{i}$ is finite but much smaller than the speed of light, $\left|\mathbf{V}_{i}\right|^{2} / c^{2} \approx 0$, we may approximate the effects of this by substituting $\mathbf{E}^{\prime}=$ $\mathbf{E}+\mathbf{V}_{i} \times \mathbf{B}$ in place of the electric field in Ohm's law. This is the transformation used in the classical theory [26]. For our purposes, this approximation should suffice, given ionic flow in ICF is distinctly nonrelativistic (e.g., the implosion velocity on the NIF point design is around $370 \mathrm{~km}^{\sim} \mathrm{s}^{-1} \sim c / 1000$ [3]).

The results here are valid for an electron-ion plasma, under the assumption of quasi-neutrality; that is, $Z n_{i}=n_{e}$. Clearly, as the temperature is increased, the effect of pair production will alter this relation to $Z n_{i}+n_{e^{+}}=n_{e^{-}}$, where the positron density $n_{e^{+}}$is a function of the optical depth of the plasma. However, in the case of the highest temperature ICF plasmas of interest (of order $100 \mathrm{keV}$ ), the positron density $n_{e^{+}}$is expected to be less than $1 \%$ of that of the electrons $n_{e^{-}}$[40], such that these results will still accurately describe electron transport. In the more general case, for which $n_{e^{+}} \lesssim n_{e^{-}}$, the kinetics of both electron and positron populations need to be considered for an accurate description of transport. This is left for further work.

Finally, our work has neglected the effect of radiative processes on the electron transport. Again this corresponds to the assumption that the plasma is optically thin; otherwise processes such as Compton scattering are likely to be significant. Irrespective of optical depth, however, bremsstrahlung may be an important consideration in collisional systems and synchrotron radiation similarly in magnetized systems. To order of magnitude, the former can be shown to be non-negligible only for temperatures $\Theta \gtrsim 10$ [10]. In order to determine the circumstances under which the latter becomes significant, consider the power radiated by an isotropic distribution of electrons in a magnetic field [41]:

$$
P=\frac{e^{4}}{9 \pi \epsilon_{0} m_{e}^{2} c^{3}} v^{2} \gamma^{2} B^{2} .
$$

(We neglect here the effect of the electric field and pressure gradients on the electron and consider solely its $\mathbf{v} \times \mathbf{B}$ rotation.)

For simplicity we confine ourselves to the ultrarelativistic limit, in which $\tau^{\star} \rightarrow 9 \Theta^{2} c^{3} / \Gamma^{e / i},\langle\gamma\rangle \rightarrow 3 \Theta$, and we can take $v \approx c$ in Eq. (63). For synchrotron processes to be negligible, we require the fractional energy loss per collision time $P \tau^{\star} /\langle\gamma\rangle m_{e} c^{2} \ll 1$ (for $\chi \ll 1$ ) or that per $\mathbf{v} \times \mathbf{B}$ rotation $P \tau^{\star} / \chi\langle\gamma\rangle m_{e} c^{2} \ll 1$ (for $\chi \gg 1$ ). Substituting the relevant parameters into the latter of these and rearranging yields

$$
\Theta Z n \ln \Lambda^{e / i} \chi \ll \frac{3}{16 \pi r_{0}^{3}},
$$

where $r_{0}=e^{2} / 4 \pi \epsilon_{0} m_{e} c^{2}$ is the classical electron radius. Taking representative values of laboratory high-energy density plasmas for the parameters on the left-hand side of this equation, e.g., $Z=1, n=1 \times 10^{32} \mathrm{~m}^{-3}$, $\ln \Lambda^{e / i}=5$, and $\chi=1$, we find a temperature condition of $\Theta \ll 5 \times 10^{9}$. Clearly this is easily satisfied, and, even though this condition is sensitive to the Hall parameter $\chi$, we find $\Theta \ll 5 \times 10^{7}$ for values as high as $\chi=100$. Beyond this, transport is no longer collisional and, as such, the process of synchrotron radiation can be safely neglected for all systems of interest.

\section{CONCLUSIONS}

In this work, a self-consistent transport theory for a relativistic plasma has been presented. It was first verified that Braginskii's transport relations [15] remain valid relativistically, in the frame in which the ions are at rest. The main system of interest, a burning ICF target, does not involve relativistic flow, and so this description should be sufficient. Transport coefficients were derived in a semianalytical form for a Lorentzian plasma $(Z \rightarrow \infty)$, which reduce to Epperlein's classical results [29] in the nonrelativistic limit. The relativistic results of other authors can also be reproduced in various limits [20,23].

For plasmas with arbitrary atomic number, the linearized Boltzmann equation was solved numerically as a means to calculate the relativistic transport coefficients. The main result of this paper is the rational fits to these, which were expressed as simple functions of the Hall parameter $\chi$ and reduced temperature $\Theta$, and reproduce the numerical results within a maximum percentage error of $20 \%$. (In most cases, particularly in the weak field limit, the error is considerably smaller than this.) To the best of our knowledge, this is the first work to provide transport coefficients for a relativistic plasma in this form. Accounting for relativistic effects results in non-negligible corrections to these coefficients, even at reasonably mild temperatures, e.g., the thermal conductivity $\kappa_{\|}$is reduced to $85 \%$ of its classical value at $k_{B} T=20 \mathrm{keV}$.

\section{ACKNOWLEDGMENTS}

The authors are grateful to M. Sherlock and E. G. Hill for helpful discussions. This work was supported by the Engineering and Physical Sciences Research Council and the Atomic Weapons Establishment, Aldermaston.

\section{APPENDIX: THE $j_{l[k] *}$ FUNCTIONS}

We catalog the $j_{l[k] *}$ functions as given by Braams and Karney [20] for $l=0,1$, where the argument $z=u / c$, the Lorentz factor $\gamma=\left(1+z^{2}\right)^{1 / 2}$, and the rapidity $\sigma=$ $\sinh ^{-1} z=\cosh ^{-1} \gamma$ :

$$
\begin{aligned}
j_{0[1] 0} & =\sigma / z, \\
j_{0[1] 1} & =1 \\
j_{0[1] 2} & =\gamma \\
j_{0[2] 02} & =(z \gamma-\sigma) / 4 z, \\
j_{0[2] 11} & =(\gamma \sigma-z) / 2 z, \\
j_{0[2] 22} & =\left[-z \gamma+\sigma\left(1+2 z^{2}\right)\right] / 8 z, \\
j_{0[3] 022} & =\left[-3 z \gamma+\sigma\left(3+2 z^{2}\right)\right] / 32 z, \\
j_{1[1] 0} & =(\gamma \sigma-z) / z^{2}, \\
j_{1[1] 1} & =(z \gamma-\sigma) / 2 z^{2}, \\
j_{1[1] 2} & =z / 3 \\
j_{1[2] 02} & =\left[-3 \gamma \sigma+3 z+z^{3}\right] / 12 z^{2}, \\
j_{1[2] 11} & =\left[-3 z \gamma+\sigma\left(3+2 z^{2}\right)\right] / 8 z^{2}, \\
j_{1[2] 22} & =\left[-\sigma \gamma\left(3-6 z^{2}\right)+3 z-5 z^{3}\right] / 72 z^{2}, \\
j_{1[3] 022} & =\left[\sigma \gamma\left(15+6 z^{2}\right)-15 z-11 z^{3}\right] / 288 z^{2} .
\end{aligned}
$$


[1] J. D. Lindl, P. Amendt, R. L. Berger, S. G. Glendinning, S. H Glenzer, S. W. Haan, R. L. Kauffman, O. L. Landen, and L. J. Suter, Phys. Plasmas 11, 339 (2004).

[2] M. J. Edwards, P. K. Patel, J. D. Lindl, L. J. Atherton, S. H. Glenzer, S. W. Haan, J. D. Kilkenny, O. L. Landen, E. I. Moses, A. Nikroo, R. Petrasso, T. C. Sangster, P. T. Springer, S. Batha, R. Benedetti, L. Bernstein, R. Betti, D. L. Bleuel, T. R. Boehly, D. K. Bradley, J. A. Caggiano, D. A. Callahan, P. M. Celliers, C. J. Cerjan, K. C. Chen, D. S. Clark, G. W. Collins, E. L. Dewald, L. Divol, S. Dixit, T. Doeppner, D. H. Edgell, J. E. Fair, M. Farrell, R. J. Fortner, J. Frenje, M. G. Gatu Johnson, E. Giraldez, V. Y. Glebov, G. Grim, B. A. Hammel, A. V. Hamza, D. R. Harding, S. P. Hatchett, N. Hein, H. W. Herrmann, D. Hicks, D. E. Hinkel, M. Hoppe, W. W. Hsing, N. Izumi, B. Jacoby, O. S. Jones, D. Kalantar, R. Kauffman, J. L. Kline, J. P. Knauer, J. A. Koch, B. J. Kozioziemski, G. Kyrala, K. N. LaFortune, S. L. Pape, R. J. Leeper, R. Lerche, T. Ma, B. J. MacGowan, A. J. MacKinnon, A. Macphee, E. R. Mapoles, M. M. Marinak, M. Mauldin, P. W. McKenty, M. Meezan, P. A. Michel, J. Milovich, J. D. Moody, M. Moran, D. H. Munro, C. L. Olson, K. Opachich, A. E. Pak, T. Parham, H. S. Park, J. E. Ralph, S. P. Regan, B. Remington, H. Rinderknecht, H. F. Robey, M. Rosen, S. Ross, J. D. Salmonson, J. Sater, D. H. Schneider, F. H. Seguin, S. M. Sepke, D. A. Shaughnessy, V. A. Smalyuk, B. K. Spears, C. Stoeckl, W. Stoeffl, L. Suter, C. A. Thomas, R. Tommasini, R. P. Town, S. V. Weber, P. J. Wegner, K. Widman, M. Wilke, D. C. Wilson, C. B. Yeamans, and A. Zylstra, Phys. Plasmas 20, 070501 (2013).

[3] J. Lindl, O. Landen, J. Edwards, E. Moses, and NIC Team, Phys. Plasmas 21, 020501 (2014).

[4] M. Tabak, J. Hammer, M. E. Glinsky, W. L. Kruer, S. C. Wilks, J. Woodworth, E. M. Campbell, M. D. Perry, and R. J. Mason, Phys. Plasmas 1, 1626 (1994).

[5] R. Betti, C. D. Zhou, K. S. Anderson, L. J. Perkins, W. Theobald, and A. A. Solodov, Phys. Rev. Lett. 98, 155001 (2007).

[6] M. Tabak, Nucl. Fusion 36, 147 (1996).

[7] S. Atzeni and J. Meyer-Ter-Vehn, The Physics of Inertial Fusion: Beam Plasma Interaction, Hydrodynamics, Hot Dense Matter (Oxford University Press, Oxford, 2004).

[8] L. J. Perkins, B. G. Logan, G. Zimmerman, and C. J. Werner, Phys. Plasmas 20, 072708 (2013).

[9] M. J.-E. Manuel, C. K. Li, F. H. Séguin, J. Frenje, D. T. Casey, R. D. Petrasso, S. X. Hu, R. Betti, J. D. Hager, D. D. Meyerhofer, and V. A. Smalyuk, Phys. Rev. Lett. 108, 255006 (2012).

[10] O. J. Pike and S. J. Rose, Phys. Rev. E 89, 053107 (2014).

[11] B. A. Trubnikov, in Reviews of Plasma Physics, edited by M. A. Leontovich (Consultants Bureau, New York, 1965), Vol. 1, p. 105.

[12] J. B. McBride and A. Pytte, Phys. Rev. 179, 145 (1969).
[13] S. T. Beliaev and G. I. Budker, Dokl. Akad. Nauk. SSSR 107, 807 (1956) [Sov. Phys. Dokl. 1, 218 (1957)].

[14] D. I. Dzhavakhishvili and N. L. Tsintsadze, Zh. Eksp. Teor. Fiz. 64, 1314 (1973) [Sov. Phys. JETP 37, 666 (1973)].

[15] S. I. Braginskii, in Reviews of Plasma Physics, edited by M. A. Leontovich (Consultants Bureau, New York, 1965), Vol. 1, p. 205.

[16] R. Balescu, I. Paiva-Veretennicoff, and L. Brenig, Physica A 81, 17 (1975).

[17] H. van Erkelens and W. A. van Leeuwen, Physica A 123, 72 (1984).

[18] G. M. Kremer and C. H. Patsko, Physica A 322, 329 (2003).

[19] B. J. Braams and C. F. F. Karney, Phys. Rev. Lett. 59, 1817 (1987).

[20] B. J. Braams and C. F. F. Karney, Phys. Fluids B 1, 1355 (1989).

[21] J. N. Mohanty and K. C. Baral, J. Phys. A 28, 5709 (1995).

[22] J. N. Mohanty and K. C. Baral, Phys. Plasmas 3, 804 (1996).

[23] M. Honda and K. Mima, J. Phys. Soc. Jpn. 67, 3420 (1998).

[24] T. Metens and R. Balescu, Phys. Fluids B 2, 2076 (1990).

[25] L. Spitzer and R. Härm, Phys. Rev. 89, 977 (1953).

[26] I. P. Shkarofsky, T. W. Johnston, and M. P. Bachynski, The Particle Kinetics of Plasmas (Addison-Wesley, Boston, 1966).

[27] M. Abramowitz and I. A. Stegun, Handbook of Mathematical Functions (Dover, New York, 1965), Chap. 9.

[28] J. L. Synge, The Relativistic Gas (North-Holland, Amsterdam, 1957).

[29] E. M. Epperlein, J. Phys. D 17, 1823 (1984).

[30] T. W. Johnston, Phys. Rev. 120, 1103 (1960).

[31] A. R. Bell, A. P. L. Robinson, M. Sherlock, R. J. Kingham, and W. Rozmus, Plasma Phys. Contr. F. 48, R37 (2006).

[32] I. P. Shkarofsky, Phys. Plasmas 4, 2464 (1997).

[33] E. M. Epperlein and M. G. Haines, Phys. Fluids 29, 1029 (1986).

[34] L. Spitzer, Jr., Physics of Fully Ionized Gases, 2nd ed. (John Wiley \& Sons, New York, 1962), Chap. 5.

[35] Our scalings here and in the preceding discussion all apply in the ultrarelativistic limit. In the mildly relativistic case, no simple scaling is possible, as the correction is a product of both the simple physical arguments here, as well as changes to the shape of the distribution function.

[36] D. R. Gray and J. D. Kilkenny, Plasma Phys. 22, 81 (1980).

[37] A. R. Bell, R. G. Evans, and D. J. Nicholas, Phys. Rev. Lett. 46, 243 (1981).

[38] J. P. Matte and J. Virmont, Phys. Rev. Lett. 49, 1936 (1982).

[39] A. R. Hochstim and G. A. Massel, in Kinetic Processes in Gases and Plasmas, edited by A. R. Hochstim (Academic Press, New York, 1969), p. 142.

[40] S. J. Rose, High Energ. Dens. Phys. 9, 480 (2013).

[41] G. B. Rybicki and A. P. Lightman, Radiative Processes in Astrophysics (John Wiley \& Sons, New York, 2008). 